\title{
Discretization and event triggered digital output feedback control of LPV systems
}

\author{
Márcio F. Braga ${ }^{\text {a }}$, Cecília F. Morais ${ }^{a}$, Eduardo S. Tognetti ${ }^{\mathrm{b}}$, Ricardo C.L.F. Oliveira ${ }^{\mathrm{a}}$, \\ Pedro L.D. Peres ${ }^{\text {a, }}$ \\ a School of Electrical and Computer Engineering, University of Campinas - UNICAMP, 13083-852, Campinas, SP, Brazil \\ ${ }^{\mathrm{b}}$ Department of Electrical Engineering, University of Brasilia - UnB, 70910-900, Brasília, DF, Brazil
}

\section{A R T I C L E I N F O}

\section{Article history:}

Received 1 December 2014

Received in revised form

11 August 2015

Accepted 12 October 2015

Available online 11 November 2015

\section{Keywords:}

Discretized linear systems

Networked control systems

Taylor series expansion

Output feedback control

Time varying parameters

Linear matrix inequalities

\begin{abstract}
A B S T R A C T
This paper investigates the problem of discretization and digital output feedback control design for continuous-time linear parameter-varying (LPV) systems subject to a time-varying networked-induced delay. The proposed discretization procedure converts a continuous-time LPV system into an equivalent discrete-time LPV system based on an extension of the Taylor series expansion and using an event-based sampling. The scheduling parameters are continuously measured and modeled as piecewise constant. A new transmission of the measured output to the controller is triggered by significant changes in the parameters, yielding time-varying transmission intervals. The obtained discretized model has matrices with polynomial dependence on the time-varying parameters and an additive norm-bounded term representing the discretization residual error. A two step strategy based on linear matrix inequality conditions is then proposed to synthesize a digital static scheduled output feedback control law that stabilizes both the discretized and the LPV model. The conditions can also be used to provide robust (i.e., independent of the scheduling parameter) static output feedback controllers. The viability of the proposed design method is illustrated through numerical examples.
\end{abstract}

(c) 2015 Elsevier B.V. All rights reserved.

\section{Introduction}

Due to technological advances, in many practical applications, the use of communication channels to implement control projects has considerably increased [1]. For instance, the exchange of data between control system components can be done by employing a networked control system (NCS) architecture. Some advantages of this framework are the use of plug-and-play devices, ease of system maintenance and diagnosis, increased system agility, and reduction in system wiring. However, despite of those benefits, some well known NCS drawbacks, like packet dropouts, multiplepacket transmission, bandwidth requirements, and networkinduced delay $[2,1]$ can restrict its use. Such disadvantages have received considerable attention from the control community, which is continuously looking for solutions to overcome the difficulties arising from the use of an NCS architecture [3-7].

In an NCS framework, the control strategy usually comprises a continuous-time plant controlled by a discrete-time controller

\footnotetext{
* Corresponding author.

E-mail address: peres@dt.fee.unicamp.br (P.L.D. Peres).
}

interfaced by analog-to-digital (A/D) and digital-to-analog (D/A) devices. This structure gives rise to two important matters that must be jointly dealt with: first, the continuous-time system can be affected by uncertainties, which occur owing to parameter variations, external perturbations, noises, the inaccuracy of sensors and actuators or related to hidden dynamics [8]; and, second, the necessity to design a digital controller that assures the stability of the closed-loop hybrid system (continuous-time plant and digital controller).

For the first problem, a possible solution is the employment of the linear parameter-varying (LPV) system theory, see $[9,10]$ and references therein. LPV modeling has increasingly evolved in the last years, mainly to represent non-linear systems in terms of a family of linear models and to describe systems whose dynamic is affected by parameters that can vary arbitrarily fast or have known bounds on the their rate of variation [11]. The second issue, generally, requires a procedure to discretize the continuoustime equations which represent the plant model (see [12] for a more detailed discussion on different ways to design digital controllers). An important observation regarding this issue is that most of the discretization approaches can only deal with plants free of uncertainties (i.e., precisely known systems) [13], or use 
approximated numerical methods that neglect the discretization error [14].

An interesting digital control approach that deals with impulsive systems and hybrid methods considering the inter-sampling behavior is presented in [15]. Following similar lines, a simplified assumption of control design for continuous-time LPV systems is employed in [16] for a class of piecewise constant parameters under constant and minimum dwell-time. Nevertheless, in the literature, there are only a few works that cope with the discretization of LPV systems. For instance, in [17,18], the authors provide conditions to design, respectively, state and output feedback digital controllers for LPV systems with desired performance specifications by employing the lifting technique [19]. Other examples can be found in $[20,21]$, where a digital controller or filter is designed through the same approach, assuming the time-varying parameters are piecewise constant. Those papers assume that both the discretization procedure and the design of the controller or filter are performed in real time, that is, the time-varying parameters are continuously read and, at each sample, a new discrete-time model related to the continuous-time plant for the current parameter is computed, and the synthesis conditions are re-evaluated. In this framework, the plant is an LTI system and the discrete-time model is exact, because, for each fixed parameter, the continuoustime system becomes precisely known. Nevertheless, this technique presents, as drawback, a large processing burden for its implementation, since the discrete-time model is computed and the design conditions are solved in real time.

This paper proposes a discretization procedure, based on an extension of the Taylor series expansion of an arbitrary degree $\ell$, which converts a continuous-time LPV model with piecewise constant parameters and a time-varying network-induced delay into an equivalent discrete-time LPV system. The accuracy of the discrete-time representation is strongly related to the increase of degree $\ell$. An event-based sampling of the output associated to the changes of the time-varying parameters is assumed. Thus, as discussed in [18], by considering the hypothesis of a time-varying sampling interval that depends on the system parameter measurements, it is possible to treat a broad class of problems, such as engines, manufacturing systems and telerobotic systems. For instance, one can cite an internal combustion engine whose sampling interval is variable and depends on the engine speed [18]. Differently from the discretization procedure proposed in [22] for uncertain time-invariant systems, the new method presented in this paper considers that the network-induced delay in the continuous-time LPV system can be time-varying. The obtained discretized model, with bounds on the rate of variation of the parameters, is described by homogeneous polynomial matrices of degree $\ell$ on the time-varying parameters, which belong to the Cartesian product of simplexes (called a multi-simplex [23]), plus a norm-bounded term related to the approximation error. The norm-bounded term depends on the degree of Taylor series expansion, the sampling time, the network-induced delay, and the original continuous-time uncertainty domain. Estimates for the bounds of the discretization residual error terms are computed through a grid in the uncertainty domain. To establish a valid discretetime LPV representation, the time-varying parameters considered in the continuous-time model are supposed piecewise constant and, therefore, the parameters do not change between two consecutive samples. Considering that the parameters are continuously monitored and have known bounds on their rate of variation, a new transmission is triggered to sample the output and the scheduled parameters whenever a significant change occurs. Otherwise, a new sample is acquired when a prescribed upper bound on the transmission interval is reached. In this scheme, the assumption that, during the sampling interval, the parameter variations are insignificant and can be neglected is valid, as considered in

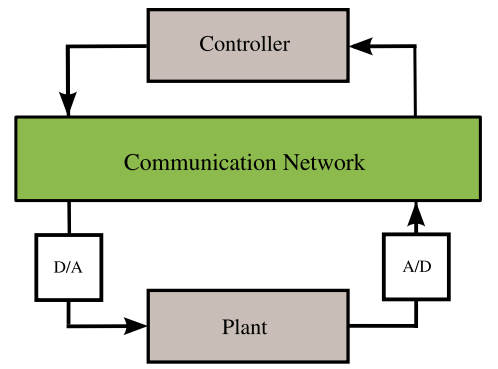

Fig. 1. Illustration of the networked control system investigated.

[16-18,20,21]. The value of the upper bound on the rate of variation of the parameters is used to specify a lower bound for the sampling period and to prevent the so-called Zeno behavior [24].

Therefore, the proposed methodology can be viewed as a parameter-based event sampling technique that can be used to deal with a wide variety of LPV systems. Compared to the usual strategy that imposes a constant small sample period to cope with abrupt variations, requiring a large bandwidth and increasing the network traffic load, the approach proposed in this paper represents an important contribution in the context of NCS.

Additionally, new conditions for state and output feedback control design of discrete-time polynomial systems with time-varying parameters are proposed in terms of linear matrix inequalities (LMIs). The conditions are solved by LMI relaxations that take into account the bounds on the rates of parameter variations. An extension of the two stage strategy [25-28] is used to provide a stabilizing static output feedback control law: initially, a parameterdependent state feedback gain is synthesized; then the outcome is applied in the second step, where a parameter-dependent output feedback controller is determined. To implement the scheduled output feedback control law, it is assumed that the time-varying parameters of the continuous-time plant can be measured or estimated in real time. If this is not the case, the conditions can also be used to determine a robust control law (parameter-independent). The use of polynomial parameter-dependent Lyapunov matrices and slack variables of arbitrary degrees in the proposed LMI relaxations can reduce the conservativeness of the synthesis conditions. The applicability of the proposed method is illustrated through numerical examples.

The remainder of the paper is structured as follows. Section 2 presents the notation, the proposed discretization technique and the event-triggered control modeling employed in this paper. Section 3 introduces the main results for the state and output feedback control design procedure. Section 4 provides numerical examples and, finally, Section 5 summarizes the paper.

\section{Problem statement}

Consider a continuous-time time-varying linear system controlled through a network communication channel by a digital controller, as illustrated in Fig. 1.

For control purposes, the following continuous-time LPV model with piecewise constant parameters is used

$$
\left\{\begin{array}{l}
\dot{x}(t)=E\left(\alpha_{1}(t)\right) x(t)+F\left(\alpha_{1}(t)\right) u(t-\tau(t)) \\
y(t)=G\left(\alpha_{1}(t)\right) x(t) \\
t \geq 0, \quad x(0)=0, \quad u(\mathcal{V})=0, \quad \mathcal{V} \in\{-\tau(t), 0\}
\end{array}\right.
$$

where $x(t) \in \mathbb{R}^{n_{x}}$ is the state space vector, $u(t) \in \mathbb{R}^{n_{u}}$ is the control signal, and $\tau(t)$ is the network-induced time-varying delay with known bounds, $\tau(t) \in\left[\tau_{1}, \tau_{2}\right]$. Matrices $E\left(\alpha_{1}(t)\right) \in$ 
$\mathbb{R}^{n_{x} \times n_{x}}, F\left(\alpha_{1}(t)\right) \in \mathbb{R}^{n_{x} \times n_{u}}$, and $G\left(\alpha_{1}(t)\right) \in \mathbb{R}^{n_{y} \times n_{x}}$ are parameterdependent and can be written as a convex combination of $N_{1}$ known vertices

$(E, F, G)\left(\alpha_{1}(t)\right)=\sum_{i=1}^{N_{1}} \alpha_{1 i}(t)\left(E_{i}, F_{i}, G_{i}\right)$

where $\alpha_{1}(t)=\left(\alpha_{11}(t), \ldots, \alpha_{1 N_{1}}(t)\right)$ is assumed to be a piecewise constant parameter vector belonging to the unit simplex, given by

$\Lambda_{N_{m}}=\left\{\left(\zeta_{1}, \ldots, \zeta_{N_{m}}\right) \in \mathbb{R}^{N_{m}}: \sum_{i=1}^{N_{m}} \zeta_{i}=1, \zeta_{i} \geq 0, i=1, \ldots, N_{m}\right\}$.

The main objective of this paper is to synthesize an output feedback digital controller that stabilizes system (1). In order to implement the digital control law, it is necessary to define a technique to sample the output signal $y(t)$ and the time varyingparameters $\alpha_{1}(t)$.

The next subsection details the sampling scheme employed in this paper.

\subsection{Event-based sampling technique}

Consider a parameter vector $\rho(t)$ of a typical LPV system that varies continuously and is measured in real-time. The absolute value of the time derivative of $\rho(t)$ is upper bounded by $\sigma>0$, that is, $\|\dot{\rho}(t)\| \leq \sigma$. Whenever a significant change of $\rho(t)$ occurs, that is, $\|\Delta \rho(t)\| \geq \epsilon$, where $\Delta \rho(t)=\rho(t)-\rho\left(t_{k}\right), t$ is the current instant, $t_{k}<t$ is the last sampling instant of $\rho(t)$, and $\epsilon>0$ is chosen by the designer, a new sample of $y(t)$ and $\rho(t)$ is sent through the network. The parameter vector $\alpha_{1}(t)$, considered in the continuous-time model (1), is defined as $\alpha_{1}(t)=\rho\left(t_{k}\right), \forall t \in$ $\left[t_{k}, t_{k+1}\right)$. Thus, by choosing a convenient value for $\epsilon$, the parameter variations during the sampling interval are insignificant and can be neglected $[17,18,20,21]$. Since $\|\dot{\rho}(t)\| \leq \sigma$, the minimum value of the elapsed time between two consecutive samples, $T\left(\alpha_{2}(t)\right)$, is given by ${ }^{1}$

$T_{\min }=\frac{\epsilon}{\sigma}$,

because

$\frac{d \rho(t)}{d t} \simeq \frac{\Delta \alpha_{1}\left(k T\left(\alpha_{2}(t)\right)\right)}{T\left(\alpha_{2}(t)\right)} \leq \frac{\epsilon}{T_{\min }}=\sigma$

where

$$
\begin{aligned}
& \Delta \alpha_{1}\left(k T\left(\alpha_{2}(t)\right)\right) \\
& \quad=\left\|\alpha_{1}\left((k+1) T\left(\alpha_{2}(t)\right)\right)-\alpha_{1}\left(k T\left(\alpha_{2}(t)\right)\right)\right\| \leq \epsilon .
\end{aligned}
$$

If no significant changes of $\rho(t)$ occurs, a new sample is triggered after time $T_{\max }$ is elapsed from the previous sample. The threshold $T_{\max }$, chosen by the designer, is related with the maximum allowable transmission interval [29]. Thus, $T\left(\alpha_{2}(t)\right)$ varies inside the interval $\left[T_{\min }, T_{\max }\right.$ ] and $\alpha_{2}(t)$ is a piecewise constant parameter belonging to the unit simplex $\Lambda_{N_{2}}, N_{2}=2$.

In this paper, $T\left(\alpha_{2}(t)\right)$ is supposed to be greater than $\tau(t)$ and, as the network-induced delay lies inside the interval $\left[\tau_{1}, \tau_{2}\right]$, it can be rewritten as $\tau\left(\alpha_{3}(t)\right)=\sum_{i=1}^{2} \alpha_{3_{i}}(t) \tau_{i}$, where $\alpha_{3}(t)$ is a piecewise constant parameter belonging to the unit simplex $\Lambda_{N_{3}}$, $N_{3}=2$. Thus $T\left(\alpha_{2}(t)\right) \geq \tau\left(\alpha_{3}(t)\right), \forall \alpha_{2}(t), \forall \alpha_{3}(t)$.

Based on these assumptions, a discretization procedure is proposed in the following subsection.

\footnotetext{
1 Note that, due to the sampling scheme adopted in this paper, the effect of chattering can be avoided, since $T_{\min }$ depends on a parameter $\epsilon$ chosen by the designer. Additionally, the Zeno Behavior phenomenon [24], i.e., the sampling period tending to zero, does not occur.
}

\subsection{Discretization procedure}

This paper proposes an equivalent discrete-time LPV model for system (1), as accurate as possible, represented by ${ }^{2}$

$$
\left\{\begin{array}{l}
x(k+1)=A(\alpha(k)) x(k)+B(\alpha(k)) u(k)+B_{d}(\alpha(k)) u(k-1) \\
y(k)=C(\alpha(k)) x(k) .
\end{array}\right.
$$

Since the time-varying parameters are considered piecewise constant they do not vary between two consecutive sampling instants, that is, $\alpha(t)=\alpha(k), \forall t \in\left[t_{k}, t_{k+1}\right)$, matrices $A(\alpha(k))$, $B(\alpha(k))$ and $B_{d}(\alpha(k))$ can be written as

$A(\alpha(k))=e^{E\left(\alpha_{1}(t)\right) T\left(\alpha_{2}(t)\right)}$

$B(\alpha(k))=\left(\int_{0}^{\gamma\left(\alpha_{2}, \alpha_{3}\right)} e^{E\left(\alpha_{1}(t)\right) s} d s\right) F\left(\alpha_{1}(t)\right)$

$B_{d}(\alpha(k))=e^{E\left(\alpha_{1}(t)\right) \Upsilon\left(\alpha_{2}, \alpha_{3}\right)}\left(\int_{0}^{\tau\left(\alpha_{3}(t)\right)} e^{E\left(\alpha_{1}(t)\right) s} d s\right) F\left(\alpha_{1}(t)\right)$,

where $\Upsilon\left(\alpha_{2}, \alpha_{3}\right)=T\left(\alpha_{2}(t)\right)-\tau\left(\alpha_{3}(t)\right)$.

The time-varying parameters affecting the system, the sampling interval and the delay can be gathered in a vector $\alpha(k)=$ $\left(\alpha_{1}(k), \alpha_{2}(k), \alpha_{3}(k)\right)$ that belongs to the multi-simplex domain $\Lambda_{N}$, given by the Cartesian product of the unit simplexes $\Lambda_{N_{m}}, m=$ $1,2,3$, as defined below.

Definition 1 (Multi-Simplex [23]). A multi-simplex $\Lambda_{N}$ is the Cartesian product $\Lambda_{N_{1}} \times \cdots \times \Lambda_{N_{r}}$ of a finite number $r$ of simplexes $\Lambda_{N_{1}}, \ldots, \Lambda_{N_{r}}$. The dimension of the $\Lambda_{N}$ is defined as the index $N=$ $\left(N_{1}, \ldots, N_{r}\right)$. For ease of notation, $\mathbb{R}^{N}$ denotes the space $\mathbb{R}^{N_{1}+\cdots+N_{r}}$. A given element $\alpha \in \Lambda_{N}$ is a vector belonging to $\mathbb{R}^{N}$ and can be decomposed as $\left(\alpha_{1}, \alpha_{2}, \ldots, \alpha_{r}\right)$ according to the structure of $\Lambda_{N}$ and, subsequently, each $\alpha_{m}$ (being in $\Lambda_{N_{m}} \subset \mathbb{R}^{N_{m}}$ ), $m=1, \ldots, r$, is decomposed in the form $\left(\alpha_{m 1}, \alpha_{m 2}, \ldots, \alpha_{m N_{m}}\right)$.

To circumvent the difficulty of dealing with the exponential of parameter-dependent matrices, a systematic procedure based on Taylor series expansion is proposed to compute, as accurate as possible, the expressions in (7). Therefore, the matrices of system (6) can be written as ${ }^{3}$

$$
\begin{aligned}
& A(\alpha)=A_{\ell}(\alpha)+\Delta A_{\ell}(\alpha), \\
& B(\alpha)=B_{\ell}(\alpha)+\Delta B_{\ell}(\alpha), \\
& B_{d}(\alpha)=B_{d \ell}(\alpha)+\Delta B_{d \ell}(\alpha) \\
& C(\alpha)=G\left(\alpha_{1}\right)
\end{aligned}
$$

with

$$
\begin{aligned}
& A_{\ell}(\alpha)=\sum_{j=0}^{\ell} \frac{E\left(\alpha_{1}\right)^{j}}{j !} T\left(\alpha_{2}\right)^{j} \\
& B_{\ell}(\alpha)=\sum_{j=1}^{\ell} \frac{E\left(\alpha_{1}\right)^{j-1}}{j !} \Upsilon\left(\alpha_{2}, \alpha_{3}\right)^{j} F\left(\alpha_{1}\right) \\
& B_{d \ell}(\alpha)=\sum_{i=0}^{\ell} \sum_{j=1}^{\ell} \frac{E\left(\alpha_{1}\right)^{i} E\left(\alpha_{1}\right)^{j-1}}{i ! j !} \Upsilon\left(\alpha_{2}, \alpha_{3}\right)^{i} \tau\left(\alpha_{3}\right)^{j} F\left(\alpha_{1}\right)
\end{aligned}
$$

and

$$
\Delta A_{\ell}(\alpha)=e^{E\left(\alpha_{1}\right) T\left(\alpha_{2}\right)}-A_{\ell}(\alpha)
$$

\footnotetext{
2 For simplicity of notation, the instant $k T\left(\alpha_{2}(t)\right)$ is denoted by $k$.

3 For simplicity of notation, the dependence of $\alpha(\cdot)$ on time is omitted hereafter
} 


$$
\begin{aligned}
\Delta B_{\ell}(\alpha) & =\left(\int_{0}^{\Upsilon\left(\alpha_{2}, \alpha_{3}\right)} e^{E\left(\alpha_{1}\right) s} d s\right) F\left(\alpha_{1}\right)-B_{\ell}(\alpha) \\
\Delta B_{d \ell}(\alpha) & =e^{E\left(\alpha_{1}\right) \Upsilon\left(\alpha_{2}, \alpha_{3}\right)}\left(\int_{0}^{\tau\left(\alpha_{3}\right)} e^{E\left(\alpha_{1}\right) s} d s\right) F\left(\alpha_{1}\right)-B_{d \ell}\left(\alpha_{1}\right)
\end{aligned}
$$

where $\Delta A_{\ell}(\alpha), \Delta B_{\ell}(\alpha)$ and $\Delta B_{d \ell}(\alpha)$ are the residues of the $\ell$-order Taylor series expansion.

Using the definitions related to $N$-tuples and multinomial series presented in A, one can write (9) as

$$
\begin{aligned}
A_{\ell}(\alpha) & =\mathrm{I}+T\left(\alpha_{2}\right) E\left(\alpha_{1}\right)+\frac{T\left(\alpha_{2}\right)^{2}}{2} E\left(\alpha_{1}\right)^{2}+\cdots+\frac{T\left(\alpha_{2}\right)^{\ell}}{\ell !} E\left(\alpha_{1}\right)^{\ell} \\
= & \sum_{s=0}^{\ell}\left(\sum_{i=1}^{N_{1}} \alpha_{1 i}\right)^{\ell-s}\left(\sum_{i=1}^{2} \alpha_{2 i}\right)^{\ell-s} \frac{T\left(\alpha_{2}\right)^{s}}{s !} E\left(\alpha_{1}\right)^{s} \\
= & \sum_{k \in \mathcal{K}_{N_{1}}(\ell) \times \mathcal{K}_{2}(\ell) \times \mathcal{K}_{2}(0)} \alpha^{k} \\
& \times\left(\sum_{j=0}^{\ell} \sum_{\substack{\hat{k} \in \mathcal{K}_{N_{1}}(\ell-j) \times \mathcal{K}_{2}(\ell-j) \times \mathcal{K}_{2}(0) \\
k \geq \hat{k} \in \mathcal{K}_{N}(j) \times, \mathcal{K}_{2}(j) \times \mathcal{K}_{2}(0) \\
k-\hat{k} \geq \tilde{k}}} \frac{((\ell-j) !)^{2}}{\hat{v} \in \mathcal{R}\left(\tilde{k}_{1}\right)} T_{\tilde{k}_{2}} E_{v}\right) \\
\triangleq & \sum_{k \in \mathcal{K}_{N_{1}}(\ell) \times \mathcal{K}_{2}(\ell) \times \mathcal{K}_{2}(0)} \alpha^{k} A_{k} \\
= & \sum_{k_{1} \in \mathcal{K}_{N_{1}}(\ell)} \sum_{k_{2} \in \mathcal{K}_{2}(\ell)} \sum_{\substack{k_{3} \in \mathcal{K}_{2}(0) \\
\alpha_{1}}} \alpha_{1}^{k_{1}} \alpha_{2}^{k_{2}} \alpha_{3}^{k_{3}} A_{k_{1} k_{2} k_{3}},
\end{aligned}
$$

matrix (10) can also be written as

$$
\begin{aligned}
& B_{\ell}(\alpha)=\Upsilon\left(\alpha_{2}, \alpha_{3}\right) F\left(\alpha_{1}\right)+\frac{\Upsilon\left(\alpha_{2}, \alpha_{3}\right)^{2}}{2} E\left(\alpha_{1}\right) F\left(\alpha_{1}\right) \\
& +\cdots+\frac{\Upsilon\left(\alpha_{2}, \alpha_{3}\right)^{\ell}}{\ell !} E\left(\alpha_{1}\right)^{\ell-1} F\left(\alpha_{1}\right) \\
& =\sum_{s=1}^{\ell}\left(\sum_{i=1}^{N_{1}} \alpha_{1 i}\right)^{\ell-s}\left(\sum_{i=1}^{2} \alpha_{2 i}\right)^{\ell-s}\left(\sum_{i=1}^{2} \alpha_{3 i}\right)^{\ell-s} \frac{\Upsilon\left(\alpha_{2}, \alpha_{3}\right)^{s}}{s !} \\
& \times E\left(\alpha_{1}\right)^{s-1} F\left(\alpha_{1}\right) \\
& =\sum_{k \in \mathcal{K}_{N}(\ell 1)} \alpha^{k}\left(\sum_{j=1}^{\ell} \sum_{\substack{\hat{k} \in \mathcal{K}_{N}((\ell-j) 1) \\
k \geq \hat{k}}} \sum_{\substack{i \in\left\{1, \ldots, N_{1}\right\} \\
k_{1}-\hat{k}_{1}-e_{i} \geq 0}} \sum_{\substack{\tilde{k} \in \mathcal{K}_{N_{1}}(j) \times \mathcal{K}_{4}(j) \\
k-\hat{k} \geq \tilde{k} \\
v \in \mathcal{R}\left(\hat{k}_{1}-e_{i}\right)}}\right. \\
& \times \frac{(-1)^{k_{3}-\hat{k}_{3}-\tilde{k}_{3}}((\ell-j) !)^{3} \sum_{i=1}^{2} \tilde{k}_{2 i} \sum_{i=1}^{2} \tilde{k}_{3 i}}{\hat{k} ! \tilde{k}_{2} ! \tilde{k}_{3} !\left(k_{2}-\hat{k}_{2}-\tilde{k}_{2}\right) !\left(k_{3}-\hat{k}_{3}-\tilde{k}_{3}\right) !} T_{k_{2}-\hat{k}_{2}-\tilde{k}_{2}} \tau_{k_{3}-\hat{k}_{3}-\tilde{k}_{3}} E_{v} F_{i} \\
& \triangleq \sum_{k_{1} \in \mathcal{K}_{N_{1}}(\ell)} \sum_{k_{2} \in \mathcal{K}_{2}(\ell)} \sum_{k_{3} \in \mathcal{K}_{2}(\ell)} \alpha^{k} B_{k} \\
& =\sum_{k_{1} \in \mathcal{K}_{N_{1}}(\ell)} \sum_{k_{2} \in \mathcal{K}_{2}(\ell)} \sum_{k_{3} \in \mathcal{K}_{2}(\ell)} \alpha_{1}^{k_{1}} \alpha_{2}^{k_{2}} \alpha_{3}^{k_{3}} B_{k_{1} k_{2} k_{3}},
\end{aligned}
$$

and, finally, (11) can also be written as Eq. (15) given in Box I, where $e_{i}$ is defined as a null vector with $i$ th component equal to one, $\mathbb{1}$ is the vector $(1,1,1)$, and $A_{k}, B_{k}$, and $B_{d k}$ are the coefficients of the discretized system polynomial matrices $A_{\ell}(\alpha), B_{\ell}(\alpha)$, and $B_{d \ell}(\alpha)$, respectively.

\subsection{Modeling of the parametric domain}

When dealing with time-varying parameters lying in the unit simplex, many researches assume that the parameters can vary arbitrarily fast. A less conservative result was proposed in [30], where the rate of variation of the parameters is supposed to be limited by an a priori known bound $b \in \mathbb{R}$, such that

$-b \leq \Delta \alpha_{m i}(k) \leq b, \quad$ for $i=1, \ldots, N_{m}, m=1,2,3$

where $\Delta \alpha_{m i}(k)=\alpha_{m i}(k+1)-\alpha_{m i}(k)$ and $b \in[0,1]$. In this paper, the value of $b$ is given by $\epsilon$ defined by (5).

Since $\alpha_{m}(k) \in \Lambda_{N_{m}}$, it is possible to prove that

$\sum_{i=1}^{N_{m}} \Delta \alpha_{m i}(k)=\sum_{i=1}^{N_{m}} \alpha_{m i}(k+1)-\sum_{i=1}^{N_{m}} \alpha_{m i}(k)=0$.

Vectors $\alpha_{m}(k)$ and $\Delta \alpha_{m}(k)$ are gathered and lifted into an augmented space, called $\gamma$-space and the region where the vector $\left(\alpha_{m}(k), \Delta \alpha_{m}(k)\right)$ assumes values can be modeled by the polytope

$$
\begin{aligned}
\Gamma_{b}= & \left\{\delta \in \mathbb{R}^{2 N_{m}}: \delta \in \operatorname{co}\left\{z^{1}, \ldots, z^{M_{m}}\right\},\right. \\
& z^{i}=\left(\begin{array}{c}
f^{i} \\
h^{i}
\end{array}\right), f^{i} \in \mathbb{R}^{N_{m}}, h^{i} \in \mathbb{R}^{N_{m}}, \\
& \sum_{j=1}^{N_{m}} h_{j}^{i}=0 \text { and } \sum_{j=1}^{N_{m}} f_{j}^{i}=1, \text { with } f_{j}^{i} \geq 0, \\
& \left.\forall j=1, \ldots, N_{m}, \forall i=1, \ldots, M_{m}\right\}
\end{aligned}
$$

defined as the convex combination of $M_{m}$ vectors $z^{i}$, where $M_{m}$ is the number of vertices of the $m$ th unit simplex in $\gamma$-space. The vectors $f^{i}$ and $h^{i}$ of the set $\Gamma_{b}$ are obtained following the lines presented in [30,31]. The following convex characterization relates $\alpha$ and $\gamma$ domains

$\left(\alpha_{m}(k), \Delta \alpha_{m}(k)\right)=\sum_{i=1}^{M_{m}}\left(\begin{array}{l}f^{i} \\ h^{i}\end{array}\right) \gamma_{m i}(k)=\left[\begin{array}{l}\mathbb{F}_{m} \\ \mathbb{H}_{m}\end{array}\right] \gamma_{m}(k)$

with $\mathbb{F}_{m}=\left[f^{1} \cdots f^{M_{m}}\right], \mathbb{H}_{m}=\left[h^{1} \cdots h^{M_{m}}\right]$ and $\gamma_{m}(k) \in \Lambda_{M_{m}}$. The time-varying parameter $\gamma(k)=\left(\gamma_{1}(k), \gamma_{2}(k), \ldots, \gamma_{r}(k)\right)$ belongs to the multi-simplex domain $\Lambda_{M}$, where $M=\left(M_{1}, M_{2}, \ldots, M_{r}\right) \in$ $\mathbb{N}^{r}$, given by the Cartesian product of the unit simplexes $\Lambda_{M_{m}}, m=$ $1, \ldots, r$.

Suppose that each time-varying parameter $\alpha_{i}(k)$ has limited variation. Then, there exists a linear relation $\alpha_{i}=\mathbb{F}_{i} \gamma_{i}$, with $\mathbb{F}_{i} \in \mathbb{R}^{N_{i} \times M_{i}}, \alpha_{i} \in \Lambda_{N_{i}}$ and $\gamma_{i} \in \Lambda_{M_{i}}$, for all $i=1, \ldots, r$. In this case, given a homogeneous polynomial matrix $R(\alpha)$ of degree $p=\left(p_{1}, p_{2}, \ldots, p_{r}\right) \in \mathbb{N}^{r}$ on variable $\alpha \in \Lambda_{N}$,

$$
\begin{aligned}
R(\alpha) & =\sum_{s \in \mathcal{K}_{N}(p)} \alpha^{s} R_{s} \\
& =\sum_{s_{1} \in \mathcal{K}_{N_{1}}\left(p_{1}\right)} \sum_{s_{2} \in \mathcal{K}_{N_{2}}\left(p_{2}\right)} \ldots \sum_{s_{r} \in \mathcal{K}_{N_{r}}\left(p_{r}\right)} \alpha_{1}^{s_{1}} \alpha_{2}^{s_{2}} \cdots \alpha_{r}^{s_{r}} R_{s_{1} s_{2} \cdots s_{r}},
\end{aligned}
$$

there exists an equivalent homogeneous polynomial

$$
\begin{aligned}
\widetilde{R}(\gamma) & =\sum_{t \in \mathcal{K}_{M}(p)} \gamma^{t} \widetilde{R}_{t} \\
& =\sum_{t_{1} \in \mathcal{K}_{M_{1}}\left(p_{1}\right)} \sum_{t_{2} \in \mathcal{K}_{M_{2}}\left(p_{2}\right)} \ldots \sum_{t_{r} \in \mathcal{K}_{M_{r}}\left(p_{r}\right)} \gamma_{1}^{t_{1}} \gamma_{2}^{t_{2}} \cdots \gamma_{r}^{t_{r}} \widetilde{R}_{t_{1} t_{2} \cdots t_{r}}
\end{aligned}
$$

of degree $p$, such that $R(\alpha) \equiv R(\mathbb{F} \gamma) \equiv \widetilde{R}(\gamma)$, with $\mathbb{F}=$ $\left(\mathbb{F}_{1}, \mathbb{F}_{2}, \ldots, \mathbb{F}_{r}\right)$. Thus, adapting for the multi-simplex domain the development presented in [31, A.2], the coefficients $\widetilde{R}_{t}$ of $\widetilde{R}(\gamma)$ can be constructed from the coefficients $R_{s}$ of $R(\alpha)$, using the following linear combination

$$
\widetilde{R}_{t}=\widetilde{R}_{t_{1} t_{2} \cdots t_{r}}=\sum_{s_{1} \in \mathcal{K}_{N_{1}}\left(p_{1}\right)} \sum_{s_{2} \in \mathcal{K}_{N_{2}}\left(p_{2}\right)} \cdots \sum_{s_{r} \in \mathcal{K}_{N_{r}}\left(p_{r}\right)}
$$




$$
\begin{aligned}
& B_{d \ell}(\alpha)=\left(\mathrm{I}+\Upsilon\left(\alpha_{2}, \alpha_{3}\right) E\left(\alpha_{1}\right)+\cdots+\frac{\Upsilon\left(\alpha_{2}, \alpha_{3}\right)^{\ell}}{\ell !} E\left(\alpha_{1}\right)^{\ell}\right)\left(\tau\left(\alpha_{3}\right) F\left(\alpha_{1}\right)+\frac{\tau\left(\alpha_{3}\right)^{2}}{2} E\left(\alpha_{1}\right) F\left(\alpha_{1}\right)+\cdots+\frac{\tau\left(\alpha_{3}\right)^{\ell}}{\ell !} E\left(\alpha_{1}\right)^{\ell-1} F\left(\alpha_{1}\right)\right) \\
& =\sum_{s=0}^{\ell} \sum_{q=1}^{\ell} \sum_{p=0}^{s}\left(\sum_{i=1}^{N_{1}} \alpha_{1 i}\right)^{2 \ell-s-q}\left(\sum_{j=1}^{2} \alpha_{2 j}\right)^{\ell-s+p}\left(\sum_{j=1}^{2} \alpha_{3 j}\right)^{2 \ell-p-q} \frac{(-1)^{p}}{p !(s-p) ! q !} T\left(\alpha_{2}\right)^{s-p} \tau\left(\alpha_{3}\right)^{p+q} E\left(\alpha_{1}\right)^{s+q-1} F\left(\alpha_{1}\right) \\
& =\sum_{k \in \mathcal{K}_{N_{1}}(2 \ell) \times \mathcal{K}_{2}(\ell) \times \mathcal{K}_{2}(2 \ell)} \alpha^{k}\left(\sum_{s=0}^{\ell} \sum_{q=1}^{\ell} \sum_{p=0}^{s} \frac{(-1)^{p}}{p !(s-p) ! q !}\right. \\
& \times \sum_{\substack{\hat{k} \in \mathcal{K}_{N_{1}}(2 \ell-s-q) \times \mathcal{K}_{2}(\ell-s+p) \times \mathcal{K}_{2}(2 \ell-p-q) \\
k \succeq \hat{k}}} \sum_{\substack{i \in\left\{1, \ldots, N_{1}\right\} \\
k_{1}-\hat{k}_{1}-e_{i} \succeq 0 \\
v \in \mathcal{R}\left(k_{1}-\hat{k}_{1}-e_{i}\right)}} \frac{\left(\sum_{i=1}^{2} k_{2_{i}}-\hat{k}_{2_{i}}\right) !\left(\sum_{i=1}^{2} k_{3_{i}}-\hat{k}_{3_{i}}\right) !}{\hat{k} !\left(k_{2}-\hat{k}_{2}\right) !\left(k_{3}-\hat{k}_{3}\right) !} \\
& \left.\times(2 \ell-s-q) !(\ell-s+p) !(2 \ell-p-q) ! T_{k-\hat{k}_{2}} \tau_{k-\hat{k}_{3}} E_{v} F_{i}\right) \\
& \triangleq \sum_{k \in \mathcal{K}_{N_{1}}(2 \ell) \times \mathcal{K}_{2}(\ell) \times \mathcal{K}_{2}(2 \ell)} \alpha^{k} B_{d k}=\sum_{k_{1} \in \mathcal{K}_{N_{1}}(2 \ell)} \sum_{k_{2} \in \mathcal{K}_{2}(\ell)} \sum_{k_{3} \in \mathcal{K}_{2}(2 \ell)} \alpha_{1}^{k_{1}} \alpha_{2}^{k_{2}} \alpha_{3}^{k_{3}} B_{d k_{1} k_{2} k_{3}}
\end{aligned}
$$

Box I.

$$
\begin{aligned}
& \times \sum_{k_{1} \in \mathcal{K}_{\mathbf{M}_{1 N_{1}}}} \sum_{\left(s_{1}\right)} \ldots \sum_{k_{2} \in \mathcal{K}_{\mathbf{M}_{2 N_{2}}}\left(s_{2}\right)} \ldots \sum_{k_{r} \in \mathcal{K}_{\mathbf{M}_{N_{N}}}\left(s_{r}\right)}\left(\prod_{i=1}^{r} \frac{s_{i} !}{k_{i} !}\right) \\
& \sum_{j=1}^{N_{1}} k_{1 j}=t_{1} \quad \sum_{j=1}^{N_{2}} k_{2 j}=t_{2} \quad \sum_{j=1}^{N_{r}} k_{r_{j}}=t_{r} \\
& \times \prod_{v=1}^{r}\left(\prod_{i=1}^{N_{v}} \prod_{j=1}^{M_{v}} \mathbb{F}_{v}(i, j)^{k_{v i j}}\right) R_{S},
\end{aligned}
$$

where the notation

$$
\sum_{\substack{\left.k_{i} \in \mathcal{K}_{\mathbf{M}_{i N_{i}}}{ }_{N_{i}}^{N_{j}} s_{i}\right) \\ \sum_{j=1} k_{i j}=t_{i}}}
$$

implies that in this summation over $k_{i} \in \mathcal{K}_{\mathbf{M}_{i N_{i}}}\left(p_{i}\right)$, only those terms should be considered for which $t_{i}=\sum_{j=1}^{N_{i}} k_{i j}$, for all $i=$ $1, \ldots, r$, where the vector $\mathbf{M}_{i N_{i}}=\left(M_{i}, M_{i}, \ldots, M_{i}\right) \in \mathbb{N}^{N_{i}}$ and the set $\mathcal{K}_{\mathbf{M}_{i N_{i}}}\left(s_{i}\right)$ denotes the Cartesian product

$$
\mathcal{K}_{\mathbf{M}_{i N_{i}}}\left(s_{i}\right)=\mathcal{K}_{M_{i}}\left(s_{i 1}\right) \times \mathcal{K}_{M_{i}}\left(s_{i 2}\right) \times \cdots \times \mathcal{K}_{M_{i}}\left(s_{i N_{i}}\right) .
$$

To simplify the LMI conditions presented in this paper, only the parameters related to the dynamic matrices of the continuoustime system are considered to have limited variation, while $\alpha_{2}(k)$ and $\alpha_{3}(k)$, associated respectively to the sampling interval and the network-induced delay, are supposed to vary arbitrarily fast. Such choices are due to: (1) the event that triggers the sampling is associated to the maximum known variation of $\rho(t)$; $(2)$ the elapsed time between two consecutive samples can vary arbitrarily inside the interval $\left[T_{\min }, T_{\max }\right]$ since, as soon as the previous sampling has occurred, $T\left(\alpha_{2}\right)$ can assume the minimum value, when $\left\|\Delta \alpha_{1}(t)\right\|=\epsilon$, or the maximum value, when the variation of $\rho(t)$ is insignificant in the interval $\Delta t<T_{\max }$; and (3) although it is possible to obtain the maximum and minimum bounds of the network-induced delay, usually, the behavior of $\tau$ between two samples cannot be easily estimated, thus, no assumptions are made about the time derivative of $\tau\left(\alpha_{3}(t)\right)$. In cases (2) and (3), as discussed in [32], the parameters in the advanced instant $k+1$ are independent of the current instant $k$ and belong to distinct simplexes, that is $\alpha_{2}(k+1)=\beta_{2}(k)$ and $\alpha_{3}(k+1)=\beta_{3}(k)$.
Therefore, the change of variables (22) can be adapted to cope with all the above cases, by introducing two new simplexes to deal with the advanced time instants of parameters $\alpha_{2}$ and $\alpha_{3}$.

$$
\begin{aligned}
& \widetilde{R}_{t}=\widetilde{R}_{t_{1} t_{2} t_{3} t_{4} t_{5}}=\sum_{s_{1} \in \mathcal{K}_{N_{1}}\left(p_{1}\right)} \sum_{s_{2} \in \mathcal{K}_{2}\left(p_{2}\right)} \sum_{s_{3} \in \mathcal{K}_{2}\left(p_{3}\right)} \sum_{s_{4} \in \mathcal{K}_{2}\left(p_{4}\right)} \sum_{s_{5} \in \mathcal{K}_{2}\left(p_{5}\right)} \\
& \times \sum_{k_{1} \in \mathcal{K}_{\mathbf{M}_{1 N_{1}}}{ }_{\left(s_{1}\right)}} \sum_{k_{2} \in \mathcal{K}_{\mathbf{M}_{22}}{ }_{\left(s_{2}\right)}} \sum_{k_{3} \in \mathcal{K}_{\mathbf{M}_{32}}\left(s_{3}\right)} \sum_{k_{r} \in \mathcal{K}_{\mathbf{M}_{42}}\left(s_{4}\right)} \\
& \sum_{j=1}^{N_{1}} k_{1 j}=t_{1} \quad \sum_{j=1}^{2} k_{2 j}=t_{2} \quad \sum_{j=1}^{2} k_{3 j}=t_{3} \quad \sum_{j=1}^{2} k_{4 j}=t_{4} \\
& \times \sum_{k_{5} \in \mathcal{K}_{\mathbf{M}_{52}}\left(s_{5}\right)} \prod_{i=1}^{5} \frac{s_{i} !}{k_{i} !} \times\left(\prod_{i=1}^{N_{1}} \prod_{j=1}^{M_{1}} \mathbb{G}(i, j)^{k_{1 i j}}\right) \\
& \sum_{j=1}^{2} k_{5 j}=t_{5} \\
& \times \prod_{v=2}^{5}\left(\prod_{i=1}^{2} \prod_{j=1}^{2} I(i, j)^{k_{v i j}}\right) R_{s}, \quad \forall t \in \mathcal{K}_{M}(p),
\end{aligned}
$$

where $I$ is an identity matrix and the argument $(i, j)$ indicates, respectively, the row and column of the matrix element. In the case where matrix $R$ depends on the current time instant $k$, the degree is given by $p=g=\left(g_{1}, g_{2}, 0, g_{3}, 0\right)$ and $\mathbb{G}=\mathbb{F}_{1}$ obtained by (19). On the other hand, when matrix $R$ depends on the advanced time instant $k+1, p=g=\left(g_{1}, 0, g_{2}, 0, g_{3}\right)$ and $\mathbb{G}=\mathbb{F}_{1}+\mathbb{H}_{1}$ with $\mathbb{F}_{1}$ and $\mathrm{H}_{1}$ computed by (19). Note that $R(\alpha)$ depends polynomially on parameters $\alpha_{1}, \alpha_{2}$, and $\alpha_{3}$ with degrees $g_{1}, g_{2}$, and $g_{3}$, respectively.

\section{Stabilization}

To design digital stabilizing controllers for system (1), consider that (6) is rewritten as an augmented discrete-time model given by (25), for $k \in \mathbb{N}$,

$$
\left\{\begin{array}{l}
z(k+1)=\mathbb{A}(\alpha) z(k)+\mathbb{B} u(k+1) \\
y(k)=\mathbb{C}(\alpha) z(k)
\end{array}\right.
$$

where $\mathbb{A}(\alpha)=\mathbb{A}_{\ell}(\alpha)+\Delta \mathbb{A}_{\ell}(\alpha)$,

$$
\mathbb{A}_{\ell}(\alpha)=\left[\begin{array}{ccc}
A_{\ell}(\alpha) & B_{\ell}(\alpha) & B_{d \ell}(\alpha) \\
0 & 0 & 0 \\
0 & I & 0
\end{array}\right],
$$



$\Delta \mathbb{A}_{\ell}(\alpha)=\left[\begin{array}{ccc}\Delta A_{\ell}(\alpha) & \Delta B_{\ell}(\alpha) & \Delta B_{d \ell}(\alpha) \\ 0 & 0 & 0 \\ 0 & 0 & 0\end{array}\right]$,
$\mathbb{B}=\left[\begin{array}{l}0 \\ I \\ 0\end{array}\right], \quad \mathbb{C}(\alpha)=\left[\begin{array}{c}C(\alpha)^{\prime} \\ 0 \\ 0\end{array}\right]^{\prime}, \quad z(k)=\left[\begin{array}{c}x(k) \\ u(k) \\ u(k-1)\end{array}\right]$.

The additive term $\Delta \mathbb{A}_{\ell}(\alpha)$ represents the discretization residual error, and can be bounded by $\left\|\Delta \mathbb{A}_{\ell}(\alpha)\right\| \leq \delta$, defined as

$$
\delta=\sup _{\alpha \in \Lambda_{N}}\left\|\Delta \mathbb{A}_{\ell}(\alpha)\right\| .
$$

The upper bound for the discretization error can be computed, for instance, using interval analysis methods [33,34], but the obtained value of $\delta$ is usually very conservative. In this paper, estimates of this bound are calculated by performing a search in a grid of values of $\alpha \in \Lambda_{N}$ (inner approximation). For the examples presented in the paper, tight outcomes have been obtained by making the grid points increasingly dense inside the domain. Although such procedure augments the computational burden, all the calculations are done off-line.

Matrix $\mathbb{A}_{\ell}(\alpha)$, with level $\ell \in \mathbb{N}$ of Taylor series expansion, has elements $A_{\ell}(\alpha), B_{\ell}(\alpha)$ and $B_{d \ell}(\alpha)$ with different degrees in $\alpha$, requiring a homogenization procedure, ${ }^{4}$

$$
\begin{aligned}
\hat{\mathbb{A}}_{\ell}(\alpha) & =\sum_{k \in \mathcal{K}_{N_{1}}(2 \ell) \times \mathcal{K}_{2}(\ell) \times \mathcal{K}_{2}(2 \ell)} \alpha^{k_{1}}\left[\begin{array}{ccc}
\mathbb{A}_{11} & \mathbb{A}_{12} & B_{d k} \\
0 & 0 & 0 \\
0 & \mathbb{I} & 0
\end{array}\right] \\
& =\sum_{k \in \mathcal{K}_{N_{1}}(2 \ell) \times \mathcal{K}_{2}(\ell) \times \mathcal{K}_{2}(2 \ell)} \alpha^{k_{1}} \mathbb{A}_{k},
\end{aligned}
$$

with

$$
\begin{aligned}
& \mathbb{A}_{11}=\sum_{\substack{\tilde{k} \in \mathcal{K}_{N_{1}}(\ell) \times \mathcal{K}_{2}(0) \times \mathcal{K}_{2}(2 \ell) \\
k \geq \tilde{k}}} \frac{\ell !(2 \ell) !}{\tilde{k} !} A_{k-\tilde{k}}, \\
& \mathbb{A}_{12}=\sum_{\substack{\tilde{k} \in \mathcal{K}_{N_{1}}(\ell) \times \mathcal{K}_{2}(0) \times \mathcal{K}_{2}(\ell) \\
k \geq \tilde{k}}} \frac{(\ell !)^{2}}{\tilde{k} !} B_{k-\tilde{k}}, \\
& \mathbb{I}=\frac{(2 \ell) ! \ell !(2 \ell) !}{k !} I .
\end{aligned}
$$

The representation of $\mathbb{C}(\alpha)$ in the multi-simplex domain is

$\mathbb{C}(\alpha)=\sum_{k \in \mathcal{K}_{N_{1}}(1) \times \mathcal{K}_{2}(0) \times \mathcal{K}_{2}(0)} \alpha^{k_{1}} \mathbb{C}_{k}$.

In some applications, the states of the system may not be available for feedback due to, for instance, high cost of implementation related to a large number of sensors or, when the states cannot be directly measured. Consequently, a more effective technique is the design of output feedback gains, which is accomplished in this paper using the two stage approach [25-28]. In this technique, firstly, a scheduled state feedback controller is synthesized as described in Section 3.1, then this gain is employed as an input in the second step presented in Section 3.2, where a parameter-dependent output feedback controller is determined.

Therefore, new LMI based conditions for synthesis of stabilizing state and output feedback controllers for polynomially parameterdependent discrete-time systems with additive norm-bounded uncertainties are proposed in the next subsections.

4 All monomials of the polynomial matrix are set to have the same degree.

\subsection{State feedback design conditions}

Consider the problem of designing a stabilizing parameterdependent state feedback control law

$u(k)=K(\alpha) z(k)$

by means of sufficient LMI conditions presented in Theorem 1 . Note that, in the two stage strategy, the parameter-dependent state feedback gain $K(\alpha)$ is employed only as an intermediate step in the search for an output feedback gain.

Theorem 1. The parameter-dependent state feedback gain $K(\alpha)=$ $Z(\alpha) Y^{-1}$, stabilizes system (25) or (6), and, consequently, (1), if there exist symmetric matrices $P_{k} \in \mathbb{R}^{\left(n_{x}+2 n_{u}\right) \times\left(n_{x}+2 n_{u}\right)}, k \in \mathcal{K}_{N}\left(g_{1}, g_{2}, g_{3}\right)$, matrices $Z_{k} \in \mathbb{R}^{n_{u} \times\left(n_{x}+2 n_{u}\right)}, k \in \mathcal{K}_{N}\left(h_{1}, h_{2}, h_{3}\right)$, and $Y \in$ $\mathbb{R}^{\left(n_{x}+2 n_{u}\right) \times\left(n_{x}+2 n_{u}\right)}$, a scalar variable $\lambda$, Pólya's relaxation degrees $d=$ $\left(d_{1}, d_{2}, d_{3}, d_{4}, d_{5}\right) \in \mathbb{N}^{5}$, and $\delta$ computed by (26), such that the following LMIs hold

$$
\begin{aligned}
& \Omega_{1}+\sum_{\substack{\bar{k} \in \mathcal{K}_{M}(w-g) \\
k \geq \bar{k}}} \Omega_{2}+\sum_{\substack{k^{\prime} \in \mathcal{K}_{M}(w-\bar{g}) \\
k \geq k^{\prime}}} \Omega_{3}+\sum_{\substack{\hat{k} \in \mathcal{K}_{M}(w-h) \\
k \geq \hat{k}}} \Omega_{4} \\
& +\sum_{\substack{\tilde{k} \in \mathcal{K}_{M}\left(w-\ell_{v}\right) \\
k \geq \bar{k}}} \Omega_{5}<0, \quad \forall k \in \mathcal{K}_{M}(w)
\end{aligned}
$$

where

$\Omega_{1}=\frac{w !}{k !}\left[\begin{array}{ccc}\lambda \delta^{2} I & \star & \star \\ 0 & -Y-Y^{\prime} & \star \\ 0 & Y & -\lambda I\end{array}\right]$

$\Omega_{2}=\frac{(w-g) !}{\bar{k} !} \operatorname{diag}\left(\begin{array}{c}0 \\ \widetilde{P}_{k-\bar{k}} \\ 0\end{array}\right)$,

$\Omega_{3}=\frac{(w-\bar{g}) !}{k^{\prime} !} \operatorname{diag}\left(\begin{array}{c}-\bar{P}_{k-k^{\prime}} \\ 0 \\ 0\end{array}\right)$,

$\Omega_{4}=\frac{(w-h) !}{\hat{k} !}\left[\begin{array}{ccc}0 & \star & \star \\ \widetilde{Z}_{k-\hat{k}}^{\prime} \mathrm{B}^{\prime} & 0 & \star \\ 0 & 0 & 0\end{array}\right]$,

$\Omega_{5}=\frac{\left(w-\ell_{v}\right) !}{\breve{k} !}\left[\begin{array}{ccc}0 & \star & \star \\ Y^{\prime} \widetilde{\mathbb{A}}_{k-\breve{k}}^{\prime} & 0 & \star \\ 0 & 0 & 0\end{array}\right]$,

$\ell_{v}=(2 \ell, \ell, 0, \ell, 0), g=\left(g_{1}, g_{2}, 0, g_{3}, 0\right), \bar{g}=\left(g_{1}, 0, g_{2}, 0, g_{3}\right)$, $h=\left(h_{1}, h_{2}, 0, h_{3}, 0\right)$ and $w=\max \left\{g, \bar{g}, h, \ell_{v}\right\}+d$. Matrices $\widetilde{\mathrm{A}}_{k}, \widetilde{Z}_{k}, \widetilde{P}_{k}$, and $\hat{P}_{k}$ are the coefficients of the homogeneous polynomial matrices $\widetilde{\mathbb{A}}(\gamma), Z(\gamma), \widetilde{P}(\gamma)$, and $\bar{P}(\gamma)$ obtained by employing change of variables (24), with, respectively, $R_{s}=\mathbb{A}_{k}, p=\ell_{v}, R_{s}=Z_{k}, p=h$, $R_{s}=P_{k}, p=g$, and $R_{s}=P_{k}, p=\bar{g}$, where $\mathbb{A}_{k}, Z_{k}$, and $P_{k}$ are the coefficients of matrices $\hat{\mathrm{A}}_{\ell}(\alpha), Z(\alpha)$, and $P(\alpha)$.

Proof. First, note that $\prod_{j=1}^{5}\left(\sum_{i=1}^{M_{j}} \gamma_{m i}\right)^{d_{j}}=1$ for any $d_{j} \in \mathbb{N}, j=$ $1, \ldots, 5$ and $m=1, \ldots, 5$. Defining the closed-loop matrix $A_{\ell}^{s f}(\gamma)=\widetilde{\mathbb{A}}_{\ell}(\gamma)+\mathbb{B} \tilde{Z}(\gamma) Y^{-1}$, and choosing

$\mathcal{Q}=\operatorname{diag}\left(-\bar{P}(\gamma)+\lambda \delta^{2} I, \widetilde{P}(\gamma),-\lambda I\right)$,

$\mathscr{B}=\left[\begin{array}{c}A_{\ell}^{s f}(\gamma) \\ -I \\ I\end{array}\right]^{\prime}, \quad \mathcal{B}^{\perp}=\left[\begin{array}{cc}I & 0 \\ A_{\ell}^{s f}(\gamma)^{\prime} & I \\ 0 & I\end{array}\right], \quad X=\left[\begin{array}{c}0 \\ Y^{\prime} \\ 0\end{array}\right]$,

where $\mathcal{B}^{\perp}$ denotes a arbitrary base for the null space of $\mathcal{B}$, one has

$Q+X \mathscr{B}+\mathscr{B}^{\prime} X^{\prime}<0$ 
which is (29) multiplied by $\gamma^{k}$, summed up for $k \in \mathcal{K}_{M}(w)$. Such conditions are equivalent to

$$
\left[\begin{array}{ccc}
\bar{P}(\gamma)-\lambda \delta^{2} I & \star & \star \\
\widetilde{P}(\gamma) A_{\ell}^{s f}(\gamma) & \widetilde{P}(\gamma) & \star \\
0 & \widetilde{P}(\gamma) & \lambda I
\end{array}\right]>0
$$

which was obtained from (30) by multiplying $\mathscr{B}^{\perp^{\prime}}$ on the left, $\mathscr{B}^{\perp}$ on the right and applying Schur's complement in the $(1,1)$ element. If (31) is verified, then the following condition, obtained by applying the change of variables (24) and Schur's complement, is also valid

$$
\begin{aligned}
& {\left[\begin{array}{cc}
P(\alpha+\Delta \alpha) & \star \\
P(\alpha) A_{\ell}^{s f}(\alpha)^{\prime} & P(\alpha)
\end{array}\right]-\lambda\left[\begin{array}{c}
\delta I \\
0
\end{array}\right]\left[\begin{array}{c}
\delta I \\
0
\end{array}\right]^{\prime}} \\
& -\lambda^{-1}\left[\begin{array}{c}
0 \\
P(\alpha)
\end{array}\right]\left[\begin{array}{c}
0 \\
P(\alpha)
\end{array}\right]^{\prime}>0 .
\end{aligned}
$$

Next, employing the following expression [35], for a given positive scalar $\lambda$,

$X Y+Y^{\prime} X^{\prime} \leq \lambda X X^{\prime}+\lambda^{-1} Y^{\prime} Y$,

and knowing that $\Delta \mathbb{A}_{\ell}(\alpha) \Delta \mathbb{A}_{\ell}(\alpha)^{\prime}<\delta^{2} I$, one obtains

$$
\left[\begin{array}{cc}
P(\alpha+\Delta \alpha) & \star \\
P(\alpha)\left(\mathbb{A}_{\ell}(\alpha)+\Delta \mathbb{A}_{\ell}(\alpha)+\mathbb{B} K\right)^{\prime} & P(\alpha)
\end{array}\right]>0
$$

Finally, multiply (34) on the left by $\mathcal{T}^{\prime}$ and on the right by $\mathcal{T}$, with $\mathcal{T}=\left[\begin{array}{cc}0 & P(\alpha+\Delta \alpha)^{-1} \\ P(\alpha)^{-1} & 0\end{array}\right]$,

to obtain

$$
\left[\begin{array}{cc}
P(\alpha)^{-1} & \star \\
P(\alpha+\Delta \alpha)^{-1}\left(\mathbb{A}_{\ell}(\alpha)+\Delta \mathbb{A}_{\ell}(\alpha)+\mathbb{B} K\right) & P(\alpha+\Delta \alpha)^{-1}
\end{array}\right]>0,
$$

or

$$
\begin{aligned}
& \left(\mathbb{A}_{\ell}(\alpha)+\Delta \mathbb{A}_{\ell}(\alpha)+\mathbb{B} K\right)^{\prime} P(\alpha+\Delta \alpha)^{-1} \\
& \quad \times\left(\mathbb{A}_{\ell}(\alpha)+\Delta \mathbb{A}_{\ell}(\alpha)+\mathbb{B} K\right)-P(\alpha)^{-1}<0,
\end{aligned}
$$

which certifies the closed-loop stability of system (25) or (6), and, consequently, of (1), by the Lyapunov function $v(x, \alpha)=$ $x^{\prime} P(\alpha)^{-1} x$.

Theorem 1 can be straightforward adapted to deal with a constant network-induced delay $\tau$, as shown below.

Corollary 1. The parameter-dependent state feedback gain $K(\alpha)=$ $Z(\alpha) Y^{-1}$, stabilizes system (25) or (6), and, consequently, (1), with a constant network-induced delay, if there exist symmetric matrices $P_{k} \in \mathbb{R}^{\left(n_{x}+2 n_{u}\right) \times\left(n_{x}+2 n_{u}\right)}, k \in \mathcal{K}_{N}\left(g_{1}, g_{2}\right)$, matrices $Z_{k} \in \mathbb{R}^{n_{u} \times\left(n_{x}+2 n_{u}\right)}$, $k \in \mathcal{K}_{N}\left(h_{1}, h_{2}\right)$, and $Y \in \mathbb{R}^{\left(n_{x}+2 n_{u}\right) \times\left(n_{x}+2 n_{u}\right)}$, a scalar variable $\lambda$, Pólya's relaxation degrees $d=\left(d_{1}, d_{2}, d_{3}\right) \in \mathbb{N}^{3}$, and $\delta$ computed by (26), such that (29) hold with $\ell_{v}=(2 \ell, \ell, 0), g=\left(g_{1}, g_{2}, 0\right)$, $\bar{g}=\left(g_{1}, 0, g_{2}\right), h=\left(h_{1}, h_{2}, 0\right)$ and $w=\max \left\{g, \bar{g}, h, \ell_{v}\right\}+d$. The matrices $\widetilde{A}_{k}, \widetilde{Z}_{k}, \widetilde{P}_{k}$, and $\hat{P}_{k}$ are the coefficients of the homogeneous polynomial matrices $\widetilde{A}(\gamma), \widetilde{Z}(\gamma), \widetilde{P}(\gamma)$, and $\bar{P}(\gamma)$ obtained by applying change of variables (24) excluding the last two simplexes, with, respectively, $R_{s}=\mathbb{A}_{k}, p=\ell_{v}, R_{s}=Z_{k}, p=h, R_{s}=P_{k}$, $p=g$, and $R_{s}=P_{k}, p=\bar{g}$, where $\mathbb{A}_{k}, Z_{k}$, and $P_{k}$ are the coefficients of matrices $\hat{\mathbb{A}}_{\ell}(\alpha), Z(\alpha)$, and $P(\alpha)$.

\subsection{Output feedback design conditions}

In the second stage, both $\alpha(k)$ and $y(k)$ are supposed sampled and a parameter-dependent output feedback control law is defined as

$u(k)=L(\alpha) y(k)=L(\alpha) \mathbb{C}(\alpha) z(k)$.

Thus, the following theorem is formulated for the output feedback stabilization of system (25) or (6), and, consequently, of (1).

Theorem 2. The static parameter dependent output feedback gain $L(\alpha)=H(\alpha)^{-1} J(\alpha)$ robustly stabilizes system (25), (6) and, consequently, (1), if there exist symmetric matrices $P_{k} \in \mathbb{R}^{\left(n_{x}+2 n_{u}\right) \times\left(n_{x}+2 n_{u}\right)}$, $k \in \mathcal{K}_{N}\left(g_{1}, g_{2}, g_{3}\right)$, matrices $H_{k} \in \mathbb{R}^{n_{u} \times n_{u}}$ and $J_{k} \in \mathbb{R}^{n_{u} \times n_{y}}, k \in$ $\mathcal{K}_{N}\left(v_{1}, v_{2}, v_{3}\right), Y_{k} \in \mathbb{R}^{\left(n_{x}+2 n_{u}\right) \times\left(n_{x}+2 n_{u}\right)}$ and $F_{k} \in \mathbb{R}^{\left(n_{x}+2 n_{u}\right) \times\left(n_{x}+2 n_{u}\right)}$, $k \in \mathcal{K}_{N}(f)$, with $f=\left(f_{1}, f_{2}, f_{3}, f_{4}, f_{5}\right) \in \mathbb{N}^{5}$, a scalar variable $\lambda$, Pólya's relaxation degrees $d=\left(d_{1}, d_{2}, d_{3}, d_{4}, d_{5}\right) \in \mathbb{N}^{5}$, given matrices $K_{k} \in \mathbb{R}^{n_{u} \times\left(n_{x}+2 n_{u}\right)}, k \in \mathcal{K}_{N}\left(h_{1}, h_{2}, h_{3}\right)$, solutions of Theorem 1 , and $\delta$ computed by (26), such that the following LMIs hold

$$
\begin{aligned}
& \frac{w !}{k !} \Omega_{1}+\sum_{\substack{k^{\prime} \in \mathcal{K}_{M}(w-v) \\
k \geq k^{\prime}}} \frac{(w-v) !}{k^{\prime} !} \Omega_{2}+\sum_{\substack{\hat{k} \in \mathcal{K}_{M}(w-g) \\
k \geq \hat{k}}} \frac{(w-g) !}{\hat{k} !} \Omega_{3} \\
& +\sum_{\substack{k \in \mathcal{K}_{M}(w-\bar{g}) \\
k \geq \dot{k}}} \frac{(w-\bar{g}) !}{\dot{k} !} \Omega_{4}+\sum_{\substack{\breve{k} \in \mathcal{K}_{M}(w-f) \\
k \geq \bar{k}}} \frac{(w-f) !}{\breve{k} !} \Omega_{5} \\
& +\sum_{\substack{k^{\prime} \in \mathcal{K}_{M}(w-v-\phi) \\
k \geq k^{\prime}}} \sum_{\substack{\tilde{k} \in \mathcal{K}(\phi) \\
k \geq k^{\prime}+\tilde{k}}} \frac{(w-v-\phi) !}{k^{\prime} !} \Omega_{6} \\
& +\sum_{k^{\prime} \in \mathcal{K}_{M}(w-v-h)} \sum_{\substack{k \in \mathcal{K}(h) \\
k \geq k^{\prime} \\
k \geq k^{\prime}+\bar{k}}} \frac{(w-v-h) !}{k^{\prime} !} \Omega_{7} \\
& +\sum_{\substack{\breve{k} \in \mathcal{K}_{M}(w-f-h) \\
k>\breve{k}}} \sum_{\substack{k \in \mathcal{K}_{M}(h) \\
k>\breve{k}+\bar{k}}} \frac{(w-f-h) !}{\breve{k} !} \Omega_{8} \\
& +\sum_{\substack{\breve{k} \mathcal{K}_{M}\left(w-f-\ell_{v}\right) \\
k \geq \breve{k}}} \sum_{\substack{\tilde{k} \in \mathcal{K}_{M}\left(\ell_{v}\right) \\
k \geq \breve{k}+\tilde{k}}} \frac{\left(w-f-\ell_{v}\right) !}{\breve{k} !} \Omega_{9}>0,
\end{aligned}
$$

$\forall k \in \mathcal{K}_{M}(w)$

where

$$
\begin{aligned}
& \Omega_{1}=\operatorname{diag}\left(-\lambda \delta^{2} I, 0,0, \lambda I\right) \text {, } \\
& \Omega_{2}=\operatorname{diag}\left(0,0,-\widetilde{H}_{k-k^{\prime}}-\widetilde{H}_{k-k^{\prime}}^{\prime}, 0\right) \text {, } \\
& \Omega_{3}=\operatorname{diag}\left(\widetilde{P}_{k-\hat{k}}, 0,0,0\right), \quad \Omega_{4}=\operatorname{diag}\left(0,-\bar{P}_{k-\dot{k}}, 0,0\right) \text {, } \\
& \Omega_{5}=\left[\begin{array}{cccc}
0 & \star & \star & \star \\
-F_{k-\breve{k}} & Y_{k-\breve{k}}+Y_{k-\breve{k}}^{\prime} & \star & \star \\
-\mathbb{B}^{\prime} F_{k-\breve{k}}^{\prime} & \mathbb{B}^{\prime} Y_{k-\breve{k}}^{\prime} & 0 & \star \\
F_{k-\breve{k}}^{\prime} & Y_{k-\breve{k}}^{\prime} & 0 & 0
\end{array}\right] \text {, } \\
& \Omega_{6}=\left[\begin{array}{cccc}
0 & \star & \star & \star \\
0 & 0 & \star & \star \\
\widetilde{J}_{k-k^{\prime}-\tilde{k}} \widetilde{\mathbb{C}}_{\tilde{k}} & 0 & 0 & \star \\
0 & 0 & 0 & 0
\end{array}\right] \text {, }
\end{aligned}
$$

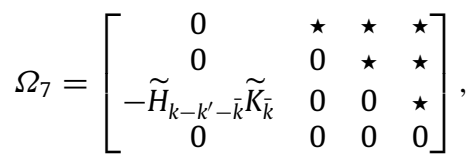

$$
\begin{aligned}
& \Omega_{8}=\left[\begin{array}{cccc}
-\widetilde{K}_{\bar{k}}^{\prime} \mathbb{B}^{\prime} F_{k-\breve{k}-\bar{k}}^{\prime}-F_{k-\breve{k}-\bar{k}} \mathbb{B} \widetilde{K}_{\bar{k}} & \star & \star & \star \\
Y_{k-\breve{k}-\bar{k}} \mathbb{B} \widetilde{K}_{\bar{k}} & 0 & \star & \star \\
0 & 0 & 0 & \star \\
0 & 0 & 0 & 0
\end{array}\right] \text {, }
\end{aligned}
$$


$\Omega_{9}=\left[\begin{array}{cccc}-\widetilde{\mathrm{A}}_{\tilde{k}}^{\prime} F_{k-\breve{k}-\tilde{k}}^{\prime}-F_{k-\breve{k}-\tilde{k}} \widetilde{\mathrm{A}}_{\tilde{k}} & \star & \star & \star \\ Y_{k-\breve{k}-\tilde{k}} \widetilde{\mathrm{A}}_{\tilde{k}} & 0 & \star & \star \\ 0 & 0 & 0 & \star \\ 0 & 0 & 0 & 0\end{array}\right]$,

$\ell_{v}=(2 \ell, \ell, 0, \ell, 0), g=\left(g_{1}, g_{2}, 0, g_{3}, 0\right), \bar{g}=\left(g_{1}, 0, g_{2}, 0, g_{3}\right)$, $h=\left(h_{1}, h_{2}, 0, h_{3}, 0\right), v=\left(v_{1}, v_{2}, 0, v_{3}, 0\right), \phi=(1,0,0,0,0)$, and $w=\max \left\{g, \bar{g}, v+\phi, f+h, h+v, f+\ell_{v}\right\}+d$. Matrices $\widetilde{\mathrm{A}}_{k}, \widetilde{\mathbb{C}}_{k}, \widetilde{H}_{k}, \widetilde{J}_{k}, \widetilde{K}_{k}, P_{k}$, and $\bar{P}_{k}$ are the coefficients of the homogeneous polynomial matrices $\widetilde{\mathbb{A}}(\gamma), \widetilde{\mathbb{C}}(\gamma), \widetilde{H}(\gamma), J(\gamma), \widetilde{K}(\gamma), \widetilde{P}(\gamma)$, and $\bar{P}(\gamma)$ obtained with change of variables (24), with, respectively, $R_{s}=\mathbb{A}_{k}$, $p=\ell_{v}, R_{s}=\mathbb{C}_{k}, p=\phi, R_{s}=H_{k}$ and $R_{s}=J_{k}$, both with $p=v$, $R_{s}=P_{k}, p=g$, and $R_{s}=P_{k}, p=\bar{g}$, where $\mathbb{A}_{k}, \mathbb{C}_{k}, H_{k}, J_{k}$, and $P_{k}$ are the coefficients of matrices $\hat{\mathrm{A}}_{\ell}(\alpha), \mathbb{C}(\alpha), H(\alpha), J(\alpha)$, and $P(\alpha)$.

Proof. First, note that with $A_{\ell}^{s f}(\gamma)=\widetilde{\mathbb{A}}_{\ell}(\gamma)+\mathbb{B} \widetilde{K}(\gamma)$, previously defined, and $A_{\ell}^{\text {of }}(\gamma)=\widetilde{\mathbb{A}}_{\ell}(\gamma)+\mathbb{B} \tilde{L}(\gamma) \widetilde{\mathbb{C}}(\gamma)$, one has

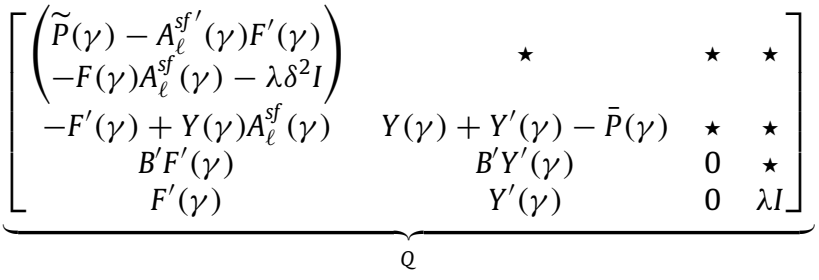

$$
\begin{aligned}
& +\underbrace{\left[\begin{array}{l}
0 \\
0 \\
I \\
0
\end{array}\right]}_{U} \underbrace{\tilde{H}(\gamma)}_{X}\left[\begin{array}{c}
S^{\prime}(\gamma) \\
0 \\
-I \\
0
\end{array}\right]^{\prime}+\underbrace{\left[\begin{array}{c}
S^{\prime}(\gamma) \\
0 \\
-I \\
0
\end{array}\right]}_{V} \tilde{H}^{\prime}(\gamma)\left[\begin{array}{l}
0 \\
0 \\
I \\
0
\end{array}\right]^{\prime}>0
\end{aligned}
$$

which is (36) multiplied by $\gamma^{k}$ and summed up for $k \in \mathcal{K}_{M}(w)$, with $S(\gamma)=\widetilde{H}(\gamma)^{-1} \widetilde{J}(\gamma) \widetilde{\mathbb{C}}(\gamma)-\widetilde{K}(\gamma)$. Choosing

$N_{V}=\left[\begin{array}{cccc}I & 0 & S(\gamma) & 0 \\ 0 & I & 0 & 0 \\ 0 & 0 & 0 & I\end{array}\right]^{\prime}, \quad N_{U}=\left[\begin{array}{cccc}I & 0 & 0 & 0 \\ 0 & I & 0 & 0 \\ 0 & 0 & 0 & I\end{array}\right]^{\prime}$,

one has that (37) is equivalent, by the Projection Lemma $[35,36]$, to

$$
\left[\begin{array}{ccc}
\widetilde{P}(\gamma)-A_{\ell}^{c l}(\gamma)^{\prime} F(\gamma)^{\prime}-F(\gamma) A_{\ell}^{c l}(\gamma)-\lambda \delta^{2} I & \star & \star \\
-F(\gamma)^{\prime}+Y(\gamma) A_{\ell}^{c l}(\gamma) & -\bar{P}(\gamma)+Y(\gamma)+Y(\gamma)^{\prime} & \star \\
F(\gamma)^{\prime} & Y(\gamma)^{\prime} & \lambda I
\end{array}\right]
$$$$
>0
$$

which is the multiplication of $Q$ in (37) by $N_{V}^{\prime}$ on the left and by $N_{V}$ on the right, replacing $A_{\ell}^{c l}(\gamma)$ in (38) by $A_{\ell}^{\text {of }}(\gamma)$, or the multiplication by $N_{U}$ on the right and on the left by its transpose, replacing $A_{\ell}^{c l}(\gamma)$ in (38) by $A_{\ell}^{s f}(\gamma)$, where the columns of $N_{V}$ and $N_{U}$ form bases of the null spaces of $V$ and $U$, respectively. By Schur's complement, (38) can be rewritten as

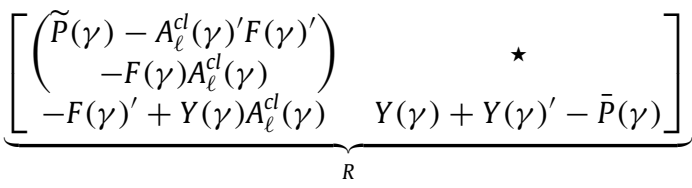

$$
\begin{aligned}
& -\lambda^{-1} \underbrace{\left[\begin{array}{l}
F(\gamma) \\
Y(\gamma)
\end{array}\right]}_{X^{\prime}}\left[\begin{array}{c}
F(\gamma) \\
Y(\gamma)
\end{array}\right]^{\prime}-\lambda\left[\begin{array}{c}
\delta I \\
0
\end{array}\right]\left[\begin{array}{c}
\delta I \\
0
\end{array}\right]^{\prime}>0 .
\end{aligned}
$$

Observing that $\Delta \mathbb{A}_{\ell}(\gamma)^{\prime} \Delta \mathbb{A}_{\ell}(\gamma)=\Delta \mathbb{A}_{\ell}(\alpha)^{\prime} \Delta \mathbb{A}_{\ell}(\alpha)<\delta^{2} I$, and by using (33), one has

$R-\left[\begin{array}{c}\Delta \mathbb{A}_{\ell}(\gamma) \\ 0\end{array}\right] X-X^{\prime}\left[\begin{array}{c}\Delta \mathbb{A}_{\ell}(\gamma) \\ 0\end{array}\right]^{\prime}>0$ which is equivalent to

$$
\begin{aligned}
& {\left[\begin{array}{cc}
\tilde{P}(\gamma)-A_{c l}(\gamma)^{\prime} F(\gamma)^{\prime}-F(\gamma) A_{c l}(\gamma) & \star \\
-F(\gamma)^{\prime}+Y(\gamma) A_{c l}(\gamma) & -\hat{P}(\gamma)+Y(\gamma)+Y(\gamma)^{\prime}
\end{array}\right]} \\
& >0
\end{aligned}
$$

where $A_{c l}(\gamma)=A_{\ell}^{c l}(\gamma)+\Delta \mathbb{A}_{\ell}(\gamma)$. Finally, multiplying (41) by $\mathscr{B}^{\prime}=\left[\begin{array}{ll}-I & A_{c l}(\gamma)^{\prime}\end{array}\right]$ on the left and by $\mathcal{B}$ on the right and, then, applying the change of variables (24), one obtains

$A_{c l}(\alpha)^{\prime} P(\alpha+\Delta \alpha) A_{c l}(\alpha)-P(\alpha)<0$

which guarantees the stability for the closed-loop system (25) or (6) with the output feedback control law (35), replacing $A_{c l}(\alpha)$ by $A_{\ell}^{\text {of }}(\alpha)+\Delta \mathbb{A}_{\ell}(\alpha)$, or with the state feedback, replacing $A_{c l}(\alpha)$ by $A_{\ell}^{s f}(\alpha)+\Delta \mathbb{A}_{\ell}(\alpha)$.

Although the stabilizability of a discrete-time LPV system, obtained from discretization procedures available in the literature, usually does not imply that the original continuous-time LPV system is also stabilizable, in the proposed approach the output feedback control law (35) guarantees the stability of the closedloop continuous-time LPV system (1), since the time-varying parameters are piecewise constant and the approximation error of the discretization procedure is taken into account. The demonstration of the previous statement is given in $\mathrm{B}$.

As done for Theorem 1, Theorem 2 can be modified to handle constant network-induced delays.

Corollary 2. The static parameter dependent output feedback gain $L(\alpha)=H(\alpha)^{-1} J(\alpha)$ robustly stabilizes system (25), (6) and, consequently, (1), with a constant network-induced delay, if there exist symmetric matrices $P_{k} \in \mathbb{R}^{\left(n_{x}+2 n_{u}\right) \times\left(n_{x}+2 n_{u}\right)}, k \in \mathcal{K}_{N}\left(g_{1}, g_{2}\right)$, matrices $H_{k} \in \mathbb{R}^{n_{u} \times n_{u}}$ and $J_{k} \in \mathbb{R}^{n_{u} \times n_{y}}, k \in \mathcal{K}_{N}\left(v_{1}, v_{2}\right), Y_{k} \in$ $\mathbb{R}^{\left(n_{x}+2 n_{u}\right) \times\left(n_{x}+2 n_{u}\right)}$ and $F_{k} \in \mathbb{R}^{\left(n_{x}+2 n_{u}\right) \times\left(n_{x}+2 n_{u}\right)}, k \in \mathcal{K}_{N}(f)$, with $f=\left(f_{1}, f_{2}, f_{3}\right) \in \mathbb{N}^{3}$, a scalar variable $\lambda$, Pólya's relaxation degrees $d=\left(d_{1}, d_{2}, d_{3}\right) \in \mathbb{N}^{3}$, given matrices $K_{k} \in \mathbb{R}^{n_{u} \times\left(n_{x}+2 n_{u}\right)}, k \in$ $\mathcal{K}_{N}\left(h_{1}, h_{2}\right)$, solutions of Corollary 1 , and $\delta$ computed by (26), such that (36) hold $\ell_{v}=(2 \ell, \ell, 0), g=\left(g_{1}, g_{2}, 0\right), \bar{g}=\left(g_{1}, 0, g_{2}\right)$, $h=\left(h_{1}, h_{2}, 0\right), v=\left(v_{1}, v_{2}, 0\right), \phi=(1,0,0)$, and $w=$ $\underset{\sim}{\max }\left\{g, \bar{g}, v+\phi, f+h, h+v, f+\ell_{v}\right\}+d$. Matrices $\widetilde{\mathrm{A}}_{k}, \widetilde{\mathbb{C}}_{k}, \widetilde{H}_{k}$, $\widetilde{J}_{k}, \widetilde{K}_{k}, P_{k}$, and $\bar{P}_{k}$ are the coefficients of the homogeneous polynomial matrices $\widetilde{\mathbb{A}}(\gamma), \widetilde{\mathbb{C}}(\gamma), \widetilde{H}(\gamma), J(\gamma), \widetilde{K}(\gamma), \widetilde{P}(\gamma)$, and $\bar{P}(\gamma)$ obtained by using change of variables (24) excluding the last two simplexes, with, respectively, $R_{s}=\mathbb{A}_{k}, p=\ell_{v}, R_{s}=\mathbb{C}_{k}, p=\phi, R_{s}=H_{k}$ and $R_{s}=J_{k}$, both with $p=v, R_{s}=P_{k}, p=g$, and $R_{s}=P_{k}, p=\bar{g}$, where $\mathbb{A}_{k}, \mathbb{C}_{k}$, $H_{k}, J_{k}$, and $P_{k}$ are the coefficients of matrices $\hat{\mathbb{A}}_{\ell}(\alpha), \mathbb{C}(\alpha), H(\alpha), J(\alpha)$, and $P(\alpha)$.

It is noteworthy to mention that, due to flexibility introduced by the multi-simplex methodology, if any time-varying parameter $\alpha_{i}, i=1,2,3$, could not be read in real time, the LMI conditions of Theorems 1 and 2 can provide parameter-independent (robust) feedback gains with respect to that parameter. In this case, it suffices to set the partial degree $h_{i}$ and $v_{i}$ equal to zero in Theorems 1 and 2, respectively.

\section{Numerical examples}

As discussed in Section 1, the existing approaches in the literature for digital control of LPV systems, which in general require the achievement of the equivalent discrete-time model and the control design to be performed in real time, do not allow a direct comparison with the proposed technique, in which the gains are previously computed and only the implementation occurs in real time. Thus, the aim of this section is to illustrate the applicability of the proposed method through numerical 
experiments. The computational effort associated to the control design conditions can be inferred by the number of scalar variables $V$ necessary to solve the set of LMIs, given in the examples. All the routines were implemented in Matlab, version 7.10 (R2010a) using Yalmip [37] and Mosek [38], in an AMD Phenom (TM) II X6 1090T (3.2 GHz), 4 GB RAM, computer.

Example 1. Consider the unstable LPV continuous-time system (1), with a time-varying network-induced delay $\tau\left(\alpha_{3}(t)\right)=$ $0.01 \alpha_{31}(t)+0.02 \alpha_{32}(t)$, whose vertices are

$$
\begin{aligned}
& E_{1}=\left[\begin{array}{cc}
0 & 1.0 \\
-2.0 & 0.3
\end{array}\right], \quad E_{2}=\beta\left[\begin{array}{cc}
0 & 1.0 \\
-1.0 & 0.5
\end{array}\right], \\
& F_{1}=\left[\begin{array}{l}
1.0 \\
2.0
\end{array}\right], \quad F_{2}=\left[\begin{array}{c}
-0.6 \\
1.0
\end{array}\right], \\
& G_{1}=\left[\begin{array}{ll}
1.0 & 1.0
\end{array}\right], \quad G_{2}=\left[\begin{array}{ll}
0 & 2.0
\end{array}\right] .
\end{aligned}
$$

The time-varying parameter $\rho(t)$ is continuously measured and satisfies $\|\dot{\rho}(t)\| \leq 6$. To guarantee that the parameter does not change significantly within one sampling interval, one chooses $\epsilon=$ 0.3 yielding $T_{\min }=0.3 / 6=0.05 \mathrm{~s}$, following (4). If the variation of $\rho(t)$ between the current instant $t$ and the previous sampling time $t_{k}$ remains lower than the preset limit $\epsilon$, that is, $\left\|\rho(t)-\rho\left(t_{k}\right)\right\| \leq \epsilon$, new samples are generated at $T_{\max }=0.1 \mathrm{~s}$. Therefore, the system is sampled with $T\left(\alpha_{2}(t)\right)=0.05 \alpha_{21}(t)+0.10 \alpha_{22}(t)$ s. Two cases are considered in this example.

\section{Case A}

In this case, $\beta$ is supposed to be equal to 0.3 and the aim is to design a scheduled output feedback digital control law that assures the closed-loop stability of the continuous-time LPV system. In practical applications, it is difficult to obtain the values of the network-induced delay in real time, so the scheduled output gain was designed to be robust with respect to $\tau\left(\alpha_{3}(t)\right)$.

For discretization levels $\ell<2$, the two stage technique does not provide feasible solutions, because the maximum bound $\delta$ for the discretization error is high. However, employing higher levels $\ell$, a more accurate dynamic description of the LPV system (42) is obtained and it is possible to synthesize a stabilizing control law. For instance, fixing $\ell=2$ in the discretization procedure and applying Theorem 1 , with $g_{i}=1, h_{i}=0, i=1,2,3$, and $d_{j}=0, j=1, \ldots, 5$, one can find a stabilizing state feedback controller by solving an LMI problem with $V=101$ scalar variables. Then, employing the state feedback gain as an input in Theorem 2, with $g_{i}=1, i=1,2,3, v_{1}=v_{2}=1, v_{3}=0$, and $f_{j}=d_{j}=0, j=1, \ldots, 5$, a parameter-dependent output feedback gain is obtained by using $V=121$ scalar variables in the LMI conditions. For this particular example, a robust (parameterindependent) output feedback control law could not be designed by Theorem 2. Fig. 2 presents a time simulation of the closed-loop behavior of the output $y(t)$, for an initial condition $x_{0}=\left[\begin{array}{ll}-3 & 2\end{array}\right]^{\prime}$, in which it is assumed that the LPV parameters $\rho(t)$ are governed by the following function

$\rho_{1}(t)=\sin ^{2}(6 t)$ and $\rho_{2}(t)=1-\rho_{1}(t)$,

and $\tau\left(\alpha_{3}(t)\right)$ has a uniform distribution inside the interval $(0.01,0.02)$. For illustration, the sampling instants are shown at the bottom of the figure.

Case B

Consider the design of stabilizing state feedback controllers by Theorem 1 for several values of $\beta>0$. The aim is to show how the conservativeness of the results, in terms of the maximum possible value of $\beta$, can be reduced by increasing the degrees of the Lyapunov matrix and of the slack variables, as shown in Table 1, that also provides the computational effort in terms of
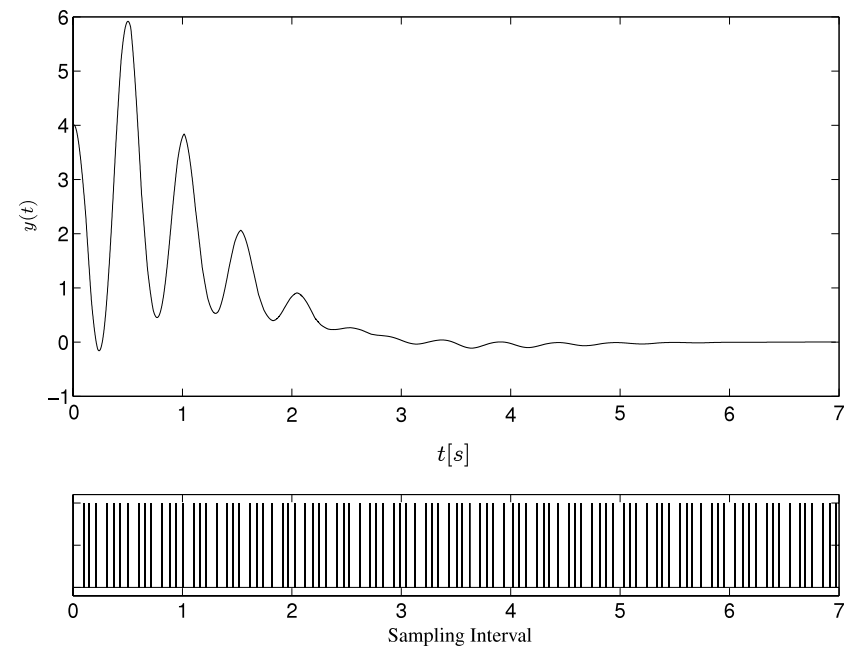

Fig. 2. Controlled output trajectories of the closed-loop system (42) with $\ell=2$, $\tau\left(\alpha_{3}(t)\right)=0.01 \alpha_{31}(t)+0.02 \alpha_{32}(t)$, and $x_{0}=\left[\begin{array}{ll}-3 & 2\end{array}\right]^{\prime}$ for Example 1.

Table 1

Upper bound of $\beta>0$ for different degrees $g$ of the Lyapunov matrix and $h$ of the slack variables in Theorem 1 for $\ell=2$ for Example 1 . To illustrate the numerical complexity, the number of scalar variables $V$ is also presented.

\begin{tabular}{lllr}
\hline$g$ & $h$ & $\beta$ & \multicolumn{1}{c}{$V$} \\
\hline$(0,0,0)$ & $(0,0,0)$ & 0.7578 & 31 \\
$(0,0,0)$ & $(1,0,0)$ & 0.9375 & 35 \\
$(1,1,1)$ & $(0,0,0)$ & 1.0781 & 101 \\
$(0,0,0)$ & $(1,1,0)$ & 1.1094 & 43 \\
$(0,0,0)$ & $(1,1,1)$ & 1.2012 & 59 \\
$(1,1,1)$ & $(1,1,1)$ & 1.4102 & 129 \\
\hline
\end{tabular}

the number of scalar variables $V$. Note that due to the flexibility of the proposed methodology, which allows to use different degrees for each decision variable and different partial degrees for each one of the unit simplexes, less conservative outcomes can be achieved with a small increase in the computational effort. For instance, analyzing the fourth row of Table 1 , one notes that the range of values of $\beta$ is expanded in approximately 3\%, using 57\% less scalar variables than the case presented in the third row of Table 1 . Additionally, by performing a more accurate discretization procedure, Theorem 1 can provide feasible solutions for a wider range of $\beta$. For instance, choosing $\ell=3$ and $g_{i}=h_{i}=0, i=$ $1,2,3$, the maximum value of $\beta$ is raised from 0.7578 (see Table 1 ) to 0.9297 .

Example 2. Consider a VTOL (Vertical Take-off and Landing) helicopter whose linearized dynamic model, adapted from [39], is described by (1) with a constant network-induced delay $\tau=$ $0.01 \mathrm{~s}$. In this model, the components $x_{1}(t), x_{2}(t), x_{3}(t), x_{4}(t)$ of the state vector $x(t)$ and $u_{1}(t), u_{2}(t)$ of the control input vector $u(t)$ represent, respectively, the horizontal and vertical velocities (knots), the pitch rate (degree/s), pitch angle (degrees), the collective pitch control and the longitudinal cyclic pitch control. The system matrices are

$E(p(t))=\left[\begin{array}{cccc}-0.0366 & 0.0271 & 0.0188 & -0.4555 \\ 0.0482 & -1.0100 & 0.0024 & -4.0208 \\ 0.1002 & p(t) & -0.7070 & 1.4200 \\ 0.0000 & 0.0000 & 1.0000 & 0.0000\end{array}\right]$,
$F_{i}=\left[\begin{array}{cc}0.4422 & 0.1761 \\ 1.4200 & -7.5922 \\ -5.5200 & 4.4900 \\ 0.0000 & 0.0000\end{array}\right]$




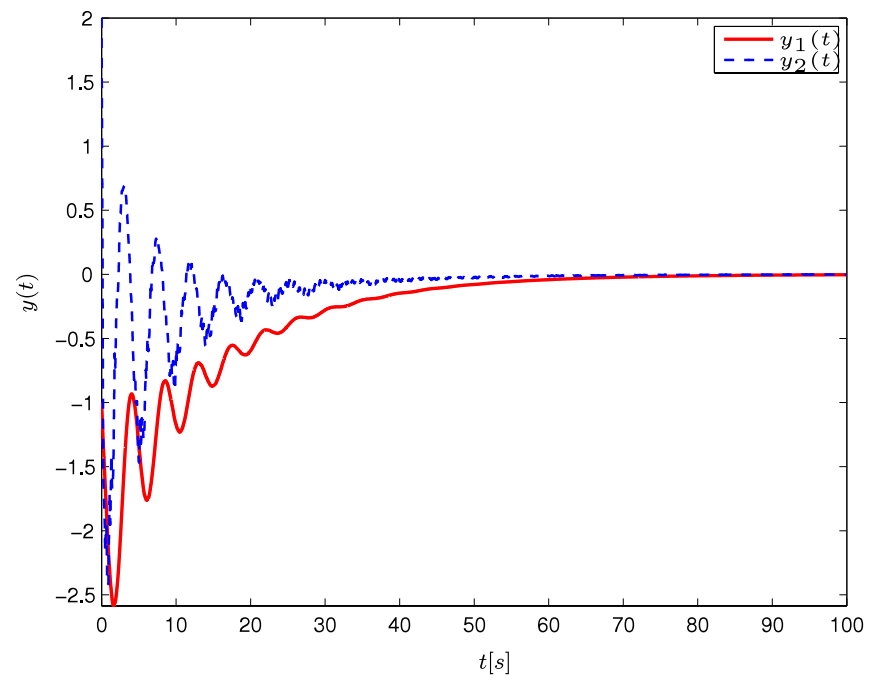

Fig. 3. Controlled output trajectories of the closed-loop VTOL system with $\ell=3$, $\tau=0.01 \mathrm{~s}$, and $x_{0}=\left[\begin{array}{llll}-1 & 2 & 1 & 3\end{array}\right]^{\prime}$ for Example 2 .

$G_{i}=\left[\begin{array}{llll}1 & 0 & 0 & 0 \\ 0 & 1 & 0 & 0\end{array}\right]$

where $p(t)=0.45+0.1319 \sin (4.56 t) \cos (9.12 t)$ and $i=1,2$. By evaluating the dynamic matrix of the system at the extreme values of the uncertain parameter $p(t) \in\left[\begin{array}{ll}0.3181 & 0.5819\end{array}\right]$, a polytope with two vertices is obtained. To convert the evolution of $p(t)$ into a parameter which belongs to a new convex parameter space domain (polytopic), one can write

$\rho_{1}(t)=\frac{1+\sin (4.56 t) \cos (9.12 t)}{2}, \quad \rho_{2}(t)=1-\rho_{1}(t)$,

and, therefore, $\rho_{1}(t), \rho_{2}(t) \in\left[\begin{array}{ll}0 & 1\end{array}\right]$. Time-varying parameter $\rho(t)$ presents a maximum time derivative of 4 , that is, $\|\dot{\rho}(t)\| \leq$ 4. To assure that parameter $\alpha_{1}(k)$ is a good piecewise constant approximation of $\rho(t), \epsilon$ in (4) is chosen equal to 0.2 yielding $T_{\min }=0.05 \mathrm{~s}$. The upper bound of the sampling interval $T_{\max }$ is adopted equal to $0.11 \mathrm{~s}$. Therefore, both horizontal and vertical velocities are measured at instants $k T\left(\alpha_{2}(t)\right)$, with $T\left(\alpha_{2}(t)\right)=$ $0.05 \alpha_{21}(t)+0.11 \alpha_{22}(t)$ s and $k=1,2, \ldots$

The aim is to design a scheduled output feedback digital controller that assures the closed-loop stability of the (open-loop unstable) continuous-time LPV system. For this purpose, state feedback gains are obtained by using the condition from Corollary 1 for the discrete-time models obtained from (13)-(15) with $\ell=$ $1,2,3$ and, then, the outcome is used as an input in Corollary 2.

For discretization levels $\ell<3$, the two stage technique does not provide feasible solutions. However, employing a more accurate discretization of the LPV system, it is possible to design a stabilizing control law. For instance, choosing $\ell=3$, the bound to the residual error computed by the discretization procedure is $\delta=1.45 \times 10^{-7}$. Then, setting $g_{i}=h_{i}=1$ and $d_{i}=0, \forall i=1,2$, in Corollary 1 , and $g_{i}=v_{i}=1, \forall i=1,2$, and $f_{j}=d_{j}=0, \forall j=$ $1,2,3$, in Corollary 2, a parameter-dependent output feedback gain is obtained. The involved number of scalar variables to solve Corollaries 1 and 2 is, respectively, 273 and 305. It is noteworthy that a stabilizing robust output feedback control law cannot be designed by Corollary 2 for this particular example. Fig. 3 presents a time simulation of the closed-loop behavior of the output $y(t)$ with the initial condition $x_{0}=\left[\begin{array}{llll}-1 & 2 & 1 & 3\end{array}\right]^{\prime}$. As can be seen, the designed scheduled controller stabilizes the continuous-time LPV system.

\section{Conclusion}

This paper presented a technique for the design of a digital scheduled output feedback controller for continuous-time LPV systems with time-varying networked-induced delay. To this end, a discretization procedure, which converts a continuous-time LPV system with piecewise constant parameters into an equivalent discrete-time LPV system, based on an extension of the Taylor series expansion and using an event-based sampling, is proposed. Additionally, new LMI conditions are given to provide a scheduled state or static output feedback control law for discrete-time LPV systems with polynomial dependence on the parameters and an additive norm bounded term. Numerical experiments illustrated that the discrete-time scheduled control law also assures the closed-loop stability of the continuous-time LPV system with piecewise constant parameters.

As future topics of research, the authors are investigating the use of $\delta$-operator [15], a technique that seems to be more suitable to deal with the case of fast-varying parameters, as well as under which assumptions it could be proved that the stabilization considering piecewise constant parameters implies the closedloop stability of the actual LPV system.

\section{Acknowledgments}

This work was supported by the Brazilian agencies CAPES, CNPq, FAPDF and grants 2011/08312-6 and 2014/22881-1, São Paulo Research Foundation (FAPESP). The authors wish to thank the anonymous reviewers for their comments and suggestions that helped to improve the paper.

\section{Appendix A. Notations and definitions for multinomial series}

As discussed in [22], products in multi-nomial series are noncommutative for matrix-valued polynomials, requiring suitable polynomial manipulations. As $E\left(\alpha_{1}\right) \in \mathbb{R}^{n_{x} \times n_{x}}$, one obtains

$$
\begin{aligned}
E\left(\alpha_{1}\right)^{q}=\left(\sum_{i=1}^{N_{1}} \alpha_{1 i} E_{i}\right)^{q} & =\sum_{p \in \mathcal{P}(q)} \prod_{i=1}^{q} \alpha_{1 p_{i}} E_{p_{i}} \\
& =\sum_{p \in \mathcal{P}(q)} \alpha_{1 p_{1}} E_{p_{1}} \cdots \alpha_{1 p_{q}} E_{p_{q}} \\
& =\sum_{p \in \mathcal{P}(q)} \alpha_{1 p} E_{p}, \quad E_{p}=E_{p_{1}} \cdots E_{p_{q}} \\
& =\sum_{k_{1} \in \mathcal{K}_{N}(q)} \alpha_{1}^{k_{1}} \sum_{p \in \mathcal{R}\left(k_{1}\right)} E_{p}
\end{aligned}
$$

where $\alpha_{1}^{k_{1}}=\alpha_{11}^{k_{11}} \alpha_{12}^{k_{12}} \cdots \alpha_{1 N_{1}}^{k_{1 N_{1}}}, k_{1}=\left(k_{11} k_{12} \cdots k_{1 N_{1}}\right), \alpha_{1 p}=$ $\left(\alpha_{1 p_{1}}, \alpha_{1 p_{2}}, \ldots, \alpha_{1 p_{q}}\right), p=\left(p_{1} p_{2} \cdots p_{q}\right)$ for $q \in \mathbb{N}, \mathcal{K}_{N_{1}}(q)$ is the set of $N_{1}$-tuples obtained as all possible combinations of non-negative integers $k_{1 i}, i=1, \ldots, N_{1}$, such that $k_{11}+k_{12}+\cdots+k_{1 N_{1}}=q$, that is

$\mathcal{K}_{N_{1}}(q) \triangleq\left\{k_{1}=\left(k_{11} k_{12} \cdots k_{1 N_{1}}\right) \in \mathbb{N}^{N_{1}}: \sum_{j=1}^{N_{1}} k_{1 j}=q, k_{1 j} \geq 0\right\}$,

$\mathcal{P}(q)$ is the set of $q$-tuples obtained as all possible combinations of non-negative integers $p_{i}, i=1, \ldots, q$, such that $p_{i} \in\left\{1, \ldots, N_{1}\right\}$, that is

$\mathcal{P}(q) \triangleq\left\{p=\left(p_{1} \cdots p_{q}\right) \in \mathbb{N}^{q}: p_{i} \in\left\{1, \ldots, N_{1}\right\}, i=1, \ldots, q\right\}$

and $\mathcal{R}\left(k_{1}\right), k_{1} \in \mathcal{K}_{N_{1}}(q)$, is the subset of all $q$-tuples $p \in \mathcal{P}(q)$ such that the elements $j$ of $p$ have multiplicity $k_{1 j}$, for $j=1, \ldots, N_{1}$, that is

$\mathcal{R}\left(k_{1}\right) \triangleq\left\{p=\left(p_{1} \cdots p_{q}\right) \in \mathbb{N}^{q}: m_{p}(j)=k_{1 j}, j=1, \ldots, N_{1}\right\}$ 
where $m_{p}(j)$ denotes the multiplicity of the element $j$ in $p$.

By definition, the set $\mathcal{K}_{N}(g)$ is the Cartesian product $K_{N}(g)=$ $\mathcal{K}_{N_{1}}\left(g_{1}\right) \times \mathcal{K}_{N_{2}}\left(g_{2}\right) \times \cdots \times \mathcal{K}_{N_{r}}\left(g_{r}\right)$, where $g=\left(g_{1}, g_{2}, \ldots, g_{r}\right) \in$ $\mathbb{N}^{r}$.

For $N$-tuples $k$ and $k^{\prime}$, one writes $k \geq k^{\prime}$ if $k_{m j} \geq k_{m j}^{\prime}, m=$ $1, \ldots, r$, and $j=1, \ldots, N_{r}$. Operations of summation $k+k^{\prime}$ and subtraction $k-k^{\prime}$ (whenever $k \geq k^{\prime}$ ) are defined component-wise. The coefficient $k$ ! is defined as

$k !=k_{11} ! k_{12} ! \cdots k_{1 N} ! \cdots k_{r 1} ! k_{r 2} ! \cdots k_{r N_{r}}$ !

where $k_{i j}$ ! stands for the factorial of $k_{i j}$, that is, $k_{i j} !=k_{i j}\left(k_{i j}-1\right) \cdots 1$.

To illustrate the definitions, consider $g=(3,1,1)$ and $N=$ $(2,2,2)$. In this case, the set $\mathcal{K}_{N}(g)$ is given by

$$
\begin{aligned}
\mathcal{K}_{N}(g)= & \mathcal{K}_{2}(3) \times \mathcal{K}_{2}(1) \times \mathcal{K}_{2}(1) \\
= & \{(30),(21),(12),(03)\} \times\{(10),(01)\} \times\{(10),(01)\} \\
= & \{(301010),(301001),(300110),(300101),(211010), \\
& (211001),(210110),(210101), \\
& (121010),(121001),(120110),(120101),(031010), \\
& (031001),(030110),(030101)\}
\end{aligned}
$$

and, for instance, choosing $k_{1}=21, k_{2}=01$, and $k_{3}=10$, $\alpha^{k}=\alpha_{1}^{k_{1}} \alpha_{2}^{k_{2}} \alpha_{3}^{k_{3}}=\alpha_{11}^{2} \alpha_{12}^{1} \alpha_{21}^{0} \alpha_{22}^{1} \alpha_{31}^{1} \alpha_{32}^{0}$. Thus, the set $\mathcal{R}\left(k_{1}\right)$ is $\mathcal{R}(21)=\{(112),(121),(211)\}$.

Finally, one obtains

$$
\sum_{p \in \mathcal{R}(21)} E_{p}=E_{1}^{2} E_{2}+E_{1} E_{2} E_{1}+E_{2} E_{1}^{2} .
$$

\section{Appendix B. Demonstration of closed-loop continuous-time LPV system stability}

The results of this paper are obtained under the hypothesis that, in the continuous-time representation of the LPV system, the time-varying parameters are piecewise constant, i.e., $\alpha(t)=$ $\alpha\left(k T\left(\alpha_{2}\right)\right) \in \Lambda_{N}, \forall t \in\left[k T\left(\alpha_{2}\right),(k+1) T\left(\alpha_{2}\right)\right)$. Since the parameter $\alpha\left(k T\left(\alpha_{2}\right)\right)$ is fixed inside the sampling interval, its dependence with respect to time can be dropped. For any $\alpha \in \Lambda_{N}$ and a given sampling period $T\left(\alpha_{2}\right)$, the solution of the linear system (1) over the interval $t \in\left[k T\left(\alpha_{2}\right),(k+1) T\left(\alpha_{2}\right)\right]$ is given by

$$
\begin{aligned}
y(t)= & G\left(\alpha_{1}\right) x(t)=G\left(\alpha_{1}\right) e^{E\left(\alpha_{1}\right)\left(t-k T\left(\alpha_{2}\right)\right)} x\left(k T\left(\alpha_{2}\right)\right) \\
& +G\left(\alpha_{1}\right) \int_{k T\left(\alpha_{2}\right)}^{t} e^{E\left(\alpha_{1}\right)(t-\hat{s})} F\left(\alpha_{1}\right) u\left(\hat{s}-\tau\left(\alpha_{3}\right)\right) d \hat{s} .
\end{aligned}
$$

Assuming that signal $u(t)$ is piecewise constant over the sampling interval, the delayed signal $u\left(t-\tau\left(\alpha_{3}\right)\right)$ is also piecewise constant. Since the delayed signal varies between two consecutive samples, one can split the integration interval of (46) in two parts such that $u\left(t-\tau\left(\alpha_{3}\right)\right)$ is constant in each part and, consequently, it can be placed outside the integral. Hence,

$$
\begin{aligned}
y(t)= & G\left(\alpha_{1}\right)\left(e^{E\left(\alpha_{1}\right)\left(t-k T\left(\alpha_{2}\right)\right)} x\left(k T\left(\alpha_{2}\right)\right)\right. \\
& +\left(\int_{k T\left(\alpha_{2}\right)+\tau\left(\alpha_{3}\right)}^{t} e^{E\left(\alpha_{1}\right)(t-\hat{s})} d \hat{s}\right) F\left(\alpha_{1}\right) u\left(k T\left(\alpha_{2}\right)\right) \\
& \left.+\left(\int_{k T\left(\alpha_{2}\right)}^{k T\left(\alpha_{2}\right)+\tau\left(\alpha_{3}\right)} e^{E\left(\alpha_{1}\right)(t-\hat{s})} d \hat{s}\right) F\left(\alpha_{1}\right) u\left((k-1) T\left(\alpha_{2}\right)\right)\right) .
\end{aligned}
$$

After performing some changes of variables, the above expression can be rewritten as

$y(t)=G\left(\alpha_{1}\right)\left(e^{E\left(\alpha_{1}\right)\left(t-k T\left(\alpha_{2}\right)\right)} x\left(k T\left(\alpha_{2}\right)\right)\right.$

$$
\begin{aligned}
& +\left(e^{E\left(\alpha_{1}\right)\left(t-k T\left(\alpha_{2}\right)-\tau\left(\alpha_{3}\right)\right)} \int_{0}^{\tau\left(\alpha_{3}\right)} e^{E\left(\alpha_{1}\right) s} d s\right) \\
& \times F\left(\alpha_{1}\right) u\left((k-1) T\left(\alpha_{2}\right)\right) \\
& +\left(e^{E\left(\alpha_{1}\right)\left(t-(k-1) T\left(\alpha_{2}\right)\right)} \int_{(k+1) T\left(\alpha_{2}\right)-t}^{T\left(\alpha_{2}\right)-\tau\left(\alpha_{3}\right)} e^{E\left(\alpha_{1}\right) s} d s\right) \\
& \left.\times F\left(\alpha_{1}\right) u\left(k T\left(\alpha_{2}\right)\right)\right) .
\end{aligned}
$$

Then, taking the supreme and using the triangle inequality, one has

$$
\begin{aligned}
& \sup _{t \in\left[k T\left(\alpha_{2}\right),(k+1) T\left(\alpha_{2}\right)\right]}\|y(t)\| \leq\left\|G\left(\alpha_{1}\right)\right\|\left(\left\|e^{E\left(\alpha_{1}\right) T\left(\alpha_{2}\right)}\right\|\left\|x\left(k T\left(\alpha_{2}\right)\right)\right\|\right. \\
& +\left\|\left(e^{E\left(\alpha_{1}\right)\left(T\left(\alpha_{2}\right)-\tau\left(\alpha_{3}\right)\right)} \int_{0}^{\tau\left(\alpha_{3}\right)} e^{E\left(\alpha_{1}\right) s} d s\right) F\left(\alpha_{1}\right)\right\| \\
& \times\left\|u\left((k-1) T\left(\alpha_{2}\right)\right)\right\| \\
& \left.+\left\|\left(\int_{0}^{T\left(\alpha_{2}\right)-\tau\left(\alpha_{3}\right)} e^{E\left(\alpha_{1}\right) s} d s\right) F\left(\alpha_{1}\right)\right\|\left\|u\left(k T\left(\alpha_{2}\right)\right)\right\|\right) .
\end{aligned}
$$

Finally, replacing (7) and (8) in (47), one has

$$
\begin{aligned}
& \sup _{t \in\left[k T\left(\alpha_{2}\right),(k+1) T\left(\alpha_{2}\right)\right]}\|y(t)\| \leq\|C(\alpha)\|\left(\left\|A_{\ell}(\alpha)+\Delta A_{\ell}(\alpha)\right\|\right. \\
& \times\left\|x\left(k T\left(\alpha_{2}\right)\right)\right\|+\left\|B_{d \ell}(\alpha)+\Delta B_{d \ell}(\alpha)\right\|\left\|u\left((k-1) T\left(\alpha_{2}\right)\right)\right\| \\
& \left.+\left\|B_{\ell}(\alpha)+\Delta B_{\ell}(\alpha)\right\|\left\|u\left(k T\left(\alpha_{2}\right)\right)\right\|\right) .
\end{aligned}
$$

Knowing that the discrete-time system (6) is closed-loop stable, i.e., $x\left(k T\left(\alpha_{2}\right)\right), u\left((k-1) T\left(\alpha_{2}\right)\right)$ and $u\left(k T\left(\alpha_{2}\right)\right)$ converge to zero as $k \rightarrow \infty$, then $y(t) \rightarrow 0$ as $t \rightarrow \infty$ and the asymptotic closedloop stability of the uncertain system (1) with the control law (35) is assured.

\section{References}

[1] W. Zhang, M.S. Branicky, S.M. Phillips, Stability of networked control systems IEEE Control Syst. Mag. 21 (1) (2001) 84-99.

[2] R.A. Borges, R.C.L.F Oliveira, C.T. Abdallah, P.L.D. Peres, th filtering for discrete-time linear systems with bounded time-varying parameters, Signal Process. 90 (1) (2010) 282-291.

[3] L. Hetel, J. Daafouz, C. Iung, Analysis and control of LTI and switched systems in digital loops via an event-based modelling, Internat. J. Control 81 (7) (2008) $1125-1138$

[4] M. Tabbara, A. Rantzer, D. Nešić, On controller \& capacity allocation co-design for networked control systems, Systems Control Lett. 58 (9)(2009) 672-676.

[5] D. Wu, J. Wu, S. Chen, Robust stabilisation control for discrete-time networked control systems, Internat. J. Control 83 (9) (2010) 1885-1894.

[6] A. Seuret, J.M. Gomes Da Silva Jr., Taking into account period variations and actuator saturation in sampled-data systems, Systems Control Lett. 61 (12) (2012) 1286-1293.

[7] E. Garcia, P.J. Antsaklis, Output feedback networked control with persistent disturbance attenuation, Systems Control Lett. 62 (10) (2013) 943-948.

[8] J. Ackermann, Robust Control: Systems with Uncertain Physical Parameters, Springer Verlag, London, UK, 1993.

[9] J. Mohammadpour, C.W. Scherer (Eds.), Control of Linear Parameter Varying Systems with Applications, Springer, New York, 2012.

[10] C. Briat, Linear Parameter-Varying and Time-Delay Systems-Analysis, Observation, Filtering and Control, in: Advances in Delays and Dynamics, vol. 3 Springer-Verlag, Berlin, Heidelberg, 2015.

[11] W.J. Rugh, J.S. Shamma, Research on gain scheduling, Automatica 36 (10 (2000) 1401-1425.

[12] S. Hara, Y. Yamamoto, H. Fujioka, Modern and classical analysis/synthesis methods in sampled-data control-a brief overview with numerical examples, in: Proceedings of the 35th IEEE Conference on Decision and Control, Kobe, Japan, December 1996, pp. 1251-1256.

[13] D.L. Gaudette, D.E. Miller, Stabilizing a first order system with an arbitrarily large time varying delay and an uncertain gain, Systems Control Lett. 62 (10) (2013) 915-923.

[14] P. Apkarian, On the discretization of LMI-synthesized linear parametervarying controllers, Automatica 33 (4) (1997) 655-661. 
[15] C. Briat, Convex conditions for robust stability analysis and stabilization of linear aperiodic impulsive and sampled-data systems under dwell-time constraints, Automatica 49 (11) (2013) 3449-3457.

[16] C. Briat, Stability analysis and control of a class of LPV systems with piecewise constant parameters, Systems Control Lett. 82 (2015) 10-17.

[17] K. Tan, K.M. Grigoriadis, F. Wu, State-feedback control of LPV sampled-data systems, in: Proceedings of the 38th IEEE Conference on Decision and Control, Phoenix, AR, USA, vol. 4, 1999, pp. 3894-3899.

[18] K. Tan, K.M. Grigoriadis, F. Wu, Output-feedback control of LPV sampled-data systems, Internat. J. Control 75 (4) (2002) 252-264.

[19] B.A. Bamieh, J.J. Pearson, A general framework for linear periodic systems with applications to $\mathscr{H}_{\infty}$ sampled-data control, IEEE Trans. Automat. Control 37 (4) (1992) 418-435.

[20] A. Ramezanifar, J. Mohammadpour, K.M. Grigoriadis, Sampled-data control of linear parameter varying time-delay systems using state feedback, in: Proceedings of the 2013 American Control Conference, Washington, DC, USA, June 2013, pp. 6847-6852.

[21] A. Ramezanifar, J. Mohammadpour, K.M. Grigoriadis, Sampled-data $\mathscr{H}_{\infty}$ filtering for linear parameter varying systems, Int. J. Syst. Sci. 46 (3) (2015) 474-487.

[22] M.F. Braga, C.F. Morais, E.S. Tognetti, R.C.L.F. Oliveira, P.L.D. Peres, Discretisation and control of polytopic systems with uncertain sampling rates and network-induced delays, Internat. J. Control 87 (11) (2014) 2398-2411.

[23] R.C.L.F. Oliveira, P.-A. Bliman, P.L.D. Peres, Robust LMIs with parameters in multi-simplex: Existence of solutions and applications, in: Proceedings of the 47th IEEE Conference on Decision and Control, Cancun, Mexico, December 2008, pp. 2226-2231.

[24] M. Heymann, F. Lin, G. Meyer, S. Resmerita, Analysis of Zeno behaviors in hybrid systems, in: Proceedings of the 41st IEEE Conference on Decision and Control, Las Vegas, NV, USA, vol. 3, December 2002, pp. 2379-2384.

[25] D. Peaucelle, D. Arzelier, An efficient numerical solution for $\mathscr{H}_{2}$ static output feedback synthesis, in: Proceedings of the 2001 European Control Conference, Porto, Portugal, September 2001, pp. 3800-3805.

[26] D. Arzelier, D. Peaucelle, S. Salhi, Robust static output feedback stabilization for polytopic uncertain systems: Improving the guaranteed performance bound in: Proceedings of the 4th IFAC Symposium on Robust Control Design, ROCOND 2003, Milan, Italy, June 2003, pp. 425-430.

[27] D. Mehdi, E.K. Boukas, O. Bachelier, Static output feedback design for uncertain linear discrete time systems, IMA J. Math. Control Inform. 21 (1) (2004) 1-13.
[28] C.M. Agulhari, R.C.L.F. Oliveira, P.L.D. Peres, LMI relaxations for reduced-order robust $\mathscr{H}_{\infty}$ control of continuous-time uncertain linear systems, IEEE Trans. Automat. Control 57 (6) (2012) 1532-1537.

[29] W.P.M.H. Heemels, A.R. Teel, N. van de Wouw, D. Nešić, Networked control systems with communication constraints: Tradeoffs between transmission intervals, delays and performance, IEEE Trans. Automat. Control 55 (8) (2010) 1781-1796.

[30] R.C.L.F Oliveira, P.L.D. Peres, Time-varying discrete-time linear systems with bounded rates of variation: Stability analysis and control design, Automatica 45 (11) (2009) 2620-2626.

[31] J. De Caigny, J.F. Camino, R.C.L.F. Oliveira, P.L.D. Peres, J. Swevers, Gainscheduled dynamic output feedback control for discrete-time LPV systems, Internat. J. Robust Nonlinear Control 22 (5) (2012) 535-558.

[32] J. Daafouz, J. Bernussou, Parameter dependent Lyapunov functions for discrete time systems with time varying parameter uncertainties, Systems Control Lett. 43 (5) (2001) 355-359.

[33] E.P. Oppenheimer, A.N. Michel, Application of interval analysis techniques to linear systems: Part II-the interval matrix exponential function, IEEE Trans. Circuits Syst. 35 (10) (1988) 1230-1242.

[34] M. Althoff, O. Stursberg, M. Buss, Reachability analysis of linear systems with uncertain parameters and inputs, in: Proceedings of the 46th IEEE Conference on Decision and Control, New Orleans, LA, USA, December 2007, pp. 726-732.

[35] S. Boyd, L. El Ghaoui, E. Feron, V. Balakrishnan, Linear Matrix Inequalities in System and Control Theory. Philadelphia, PA: SIAM Studies in Applied Mathematics, 1994.

[36] G. Pipeleers, B. Demeulenaere, J. Swevers, L. Vandenberghe, Extended LMI characterizations for stability and performance of linear systems, Systems Control Lett. 58 (7) (2009) 510-518.

[37] J. Löfberg, YALMIP: A toolbox for modeling and optimization in MATLAB, in: Proceedings of the 2004 IEEE International Symposium on Computer Aided Control Systems Design, Taipei, Taiwan, September 2004, pp. 284-289.

[38] E.D. Andersen, K.D. Andersen, The MOSEK interior point optimizer for linear programming: An implementation of the homogeneous algorithm, in: H. Frenk, K. Roos, T. Terlaky, S. Zhang (Eds.), High Performance Optimization, in: Applied Optimization, vol. 33, Springer, US, 2000, pp. 197-232. http://www.mosek.com.

[39] L. Keel, S.P. Bhattacharyya, J.W. Howze, Robust control with structured perturbations, IEEE Trans. Automat. Control 33 (1) (1988) 68-78. 